\title{
Protein-Protected Porous Bimetallic AgPt Nanoparticles with pH-switchable Peroxidase/Catalase-Mimicking Activity
}

\author{
Mustafa Gharib, ${ }^{1,2}$ Andreas Kornowski, ${ }^{3}$ Heshmat Noei, ${ }^{4}$ Wolfgang J. Parak, ${ }^{1,5^{*}}$ \\ Indranath Chakraborty ${ }^{1 *}$ \\ ${ }^{1}$ Fachbereich Physik, Center for Hybrid Nanostructures (CHyN), Universität Hamburg, Hamburg, \\ Germany \\ ${ }^{2}$ Radiation Biology Department, Egyptian Atomic Energy Authority (EAEA), Cairo, Egypt \\ ${ }^{3}$ Fachbereich Chemie, Universität Hamburg, Hamburg, Germany \\ ${ }^{4}$ DESY NanoLab, Deutsches Elektronen-Synchrotron (DESY), Hamburg, Germany \\ ${ }^{5}$ CIC Biomagune, San Sebastian, Spain \\ *corresponding authors: wolfgang.parak@uni-hamburg.de \\ indranath.chakraborty@physik.uni-hamburg.de
}

\section{SUPPORTING INFORMATION}

\section{Index}

1. Materials

2. Synthesis of prism-shaped silver nanoparticles (Ag NPs)

3. Synthesis of porous silver-platinum bimetallic nanoparticles (AgPt NPs)

4. Characterization of Ag NP seeds, Ag NPs, and AgPt NPs

5. Determination of NP concentration

6. Colloidal stability of Ag NPs and AgPt NPs

7. Effect of $\mathrm{pH}$ on the synthesis of AgPt NPs

8. Understanding the role of BSA in the synthesis

9. Effect of Pt precursor $\left(\mathrm{H}_{2} \mathrm{PtCl}_{6}\right)$ concentration on the synthesis

10. Other bimetallic NPs using the same synthesis protocol

11. Structural changes of BSA during the synthesis of Ag NPs and AgPt NPs

12. Peroxidase-like activity of AgPt NPs

13. Catalase- like activity of AgPt NPs

14. Cargo-loading/controlled-release into/from the AgPt NPs

15. References

Note that parts of the data shown in the main manuscript are compilations from data also shown in the

Supporting

Information. 


\section{Materials}

Silver nitrate $\left(\mathrm{AgNO}_{3}, 99 \%\right)$, chloroplatinic acid hexahydrate $\left(\mathrm{H}_{2} \mathrm{PtCl}_{6} \cdot 6 \mathrm{H}_{2} \mathrm{O}\right)$, palladium chloride $\left(\mathrm{PdCl}_{2}, 99 \%\right)$, gold(III) chloride trihydrate $\left(\mathrm{HAuCl}_{4} .3 \mathrm{H}_{2} \mathrm{O}, 99 \%\right)$, sodium borohydride $\left(\mathrm{NaBH}_{4}\right.$, 99.99\%), tri-sodium citrate dihydrate (99\%), ascorbic acid (99\%), bovine serum albumin (BSA), catalase, ortho-phenylenediamine (OPD, 98\%), peroxidase from horseradish (HRP) (150 U/mg), and calcein were purchased from Sigma-Aldrich. Sodium chloride $(\mathrm{NaCl}, 99.9 \%)$ and hydrogen peroxide $\left(30 \%\right.$ in $\left.\mathrm{H}_{2} \mathrm{O}\right)$ were purchased from Roth. Phosphate-buffered saline (PBS), Dulbecco's Modified Eagle Medium (DMEM), Roswell Park Memorial Institute culture medium (RPMI), and fetal bovine serum (FBS) were purchased from ThermoFischer.

\section{Synthesis of prism-shaped silver nanoparticles (Ag NPs)}

Silver prism-shaped NPs were synthesized according to a previously reported seed-mediated approach. ${ }^{1}$ First, $10 \mathrm{~mL}$ of silver seed NPs were prepared by mixing $25 \mu \mathrm{L}$ of $\mathrm{AgNO}_{3}(100 \mathrm{mM})$ and $25 \mu \mathrm{L}$ of sodium citrate $(100 \mathrm{mM})$ in a glass vial containing $10 \mathrm{~mL}$ of MilliQ water. Then the mixture was heated to $90{ }^{\circ} \mathrm{C}$ under vigorous stirring (1500 revolutions per minute (rpm)) for 15 minutes. Then, $100 \mu \mathrm{L}$ of freshly prepared sodium borohydride $(20 \mathrm{mM})$ solution was added rapidly to the mixture. A light-yellow color appeared immediately, which confirmed the formation of the silver seed NPs. The solution was then allowed to cool down to room temperature and the magnetic stir-bar was removed, after which the solution was wrapped with aluminum foil to avoid direct light exposure and was allowed to age for $24 \mathrm{~h}$ in a fridge. The growth of Ag NPs was initiated by mixing $50 \mu \mathrm{L}$ of $\mathrm{AgNO}_{3}(100 \mathrm{mM}), 120 \mu \mathrm{L}$ of BSA $\left(50 \mathrm{mg} \mathrm{mL}^{-1}\right)$, and $300 \mu \mathrm{L}$ of asprepared Ag seed NP solutions in a glass vial containing $10 \mathrm{~mL}$ of MilliQ water under vigorous stirring (1500-2000 rpm). Then, $60 \mu \mathrm{L}$ of ascorbic acid $(100 \mathrm{mM})$ was added subsequently, after which a series of color changes from faint-yellow to deep violet was observed. The synthesis of Ag NPs was completed in 25-30 minutes and was confirmed by the appearance of the three characteristic absorption peaks of prism-shaped Ag NPs. ${ }^{2}$ The as-prepared NPs were centrifuged at $9000 \mathrm{rpm}$ for 15 minutes, washed two times with MilliQ water, and redispersed in MilliQ water for further work.

\section{Synthesis of porous silver-platinum bimetallic nanoparticles (AgPt NPs)}

Porous AgPt bimetallic nanoparticles (AgPt NP) were prepared by mixing platinum precursor with the sacrificial template of prism-shaped Ag NPs, during which a galvanic replacement reaction takes place and the preformed $\mathrm{Ag}$ template is partially oxidized by the Pt precursor followed by $\mathrm{Pt}$ atom deposition and the oxidative dissolution of $\mathrm{Ag}$ template, leaving behind porous bimetallic nanoprisms. In a typical synthesis procedure, $50 \mu \mathrm{L}$ of $25 \mathrm{mM}$ platinum precursor $\left(\mathrm{H}_{2} \mathrm{PtCl}_{6}\right)$ was mixed with $10 \mathrm{~mL}$ of purified Ag NP solution under vigorous stirring $(1500 \mathrm{rpm})$ at room temperature. The reaction is completed when the solution color changes from deep violet color to 
dark navy blue color, which takes around 15-20 minutes. The AgPt NPs were purified by two consecutive cycles of centrifugation ( $9000 \mathrm{rpm}, 15$ minutes) and washing with MilliQ water (i.e. the NPs were precipitated, the supernatant was discarded, and the NPs were redissolved in water). The final product was redispersed in MilliQ water for further work. This synthetic route was also used for the synthesis of different $\mathrm{AgM}(\mathrm{M}=\mathrm{Pd}$ and $\mathrm{Au}) \mathrm{NPs}$ by replacing the Pt precursor with Palladium(II) chloride $\left(\mathrm{PdCl}_{2}\right)$ and gold(III) chloride $\left(\mathrm{HAuCl}_{4}\right)$, respectively.

\section{Characterization of Ag NP seeds, Ag NPs, and AgPt NPs}

UV-visible (UV-vis) absorbance spectra were recorded in water using an Agilent 8453 UV-vis spectrophotometer in $1 \mathrm{~cm}$ path length quartz cuvettes at room temperature in the range from 200$1100 \mathrm{~nm}$. Dynamic light scattering (DLS) and zeta potentials measurements were carried out using a Malvern Zetasizer Nanoparticle analyzer (ZEN 3600) instrument, see Figure S1.
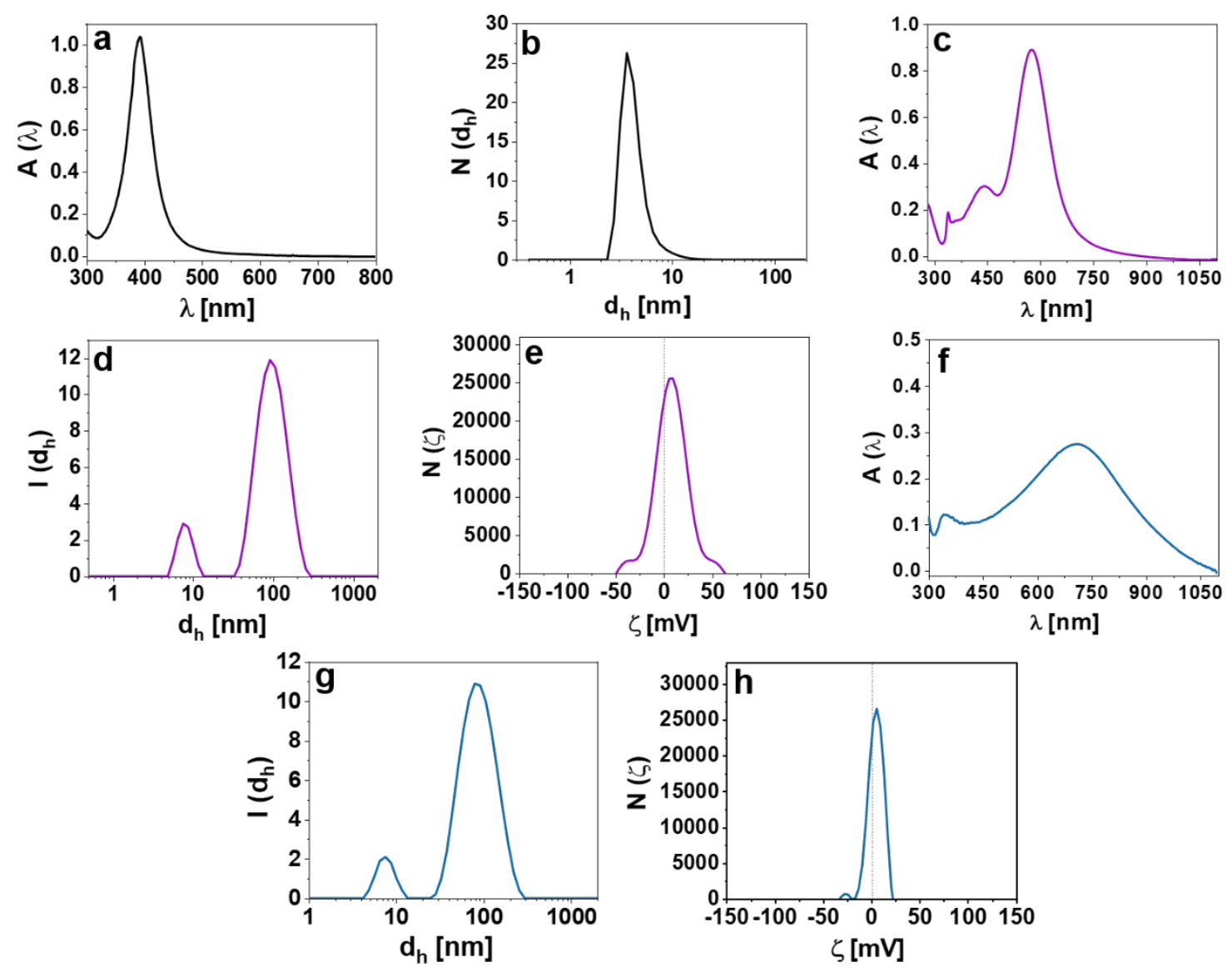

Figure S1. Characterization of Ag NP seeds, Ag NPs, and AgPt NPs. (a, c, and f) UV-vis

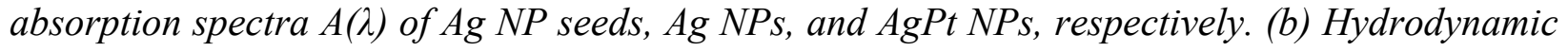
diameter of the Ag seed NPs in the form of number distribution $N\left(d_{h}\right)$. (d and $g$ ) Hydrodynamic diameter of Ag NPs and AgPt NPs, respectively, in terms of intensity distribution I $\left(d_{h}\right)$. (e and $h$ ) Zeta potential $\zeta$ of Ag NPs and AgPt NPs, respectively. 
The morphology of the as-prepared NPs was analyzed using a field emission gun JEM 2100F UHR (JEOL, Japan) transmission electron microscope (TEM), equipped with high angle annular dark field (HAADF). The samples were prepared by drop-casting the NP dispersions on ultrathin carbon-coated copper grids (400-mesh). Extra solution was removed by a blotting filter paper and then the grids were left to dry at room temperature (Figures S2 and S3). Elemental distribution of $\mathrm{Ag}$ and Pt within the NPs was detected by energy-dispersive X-ray spectroscopy (EDS) spot analysis through a Philips CM 300 UT (LaB6) TEM operating at $200 \mathrm{kV}$, equipped with SDD EDX detector (Ametek) and CCD camera (Gatan) (cf. Figure 1).
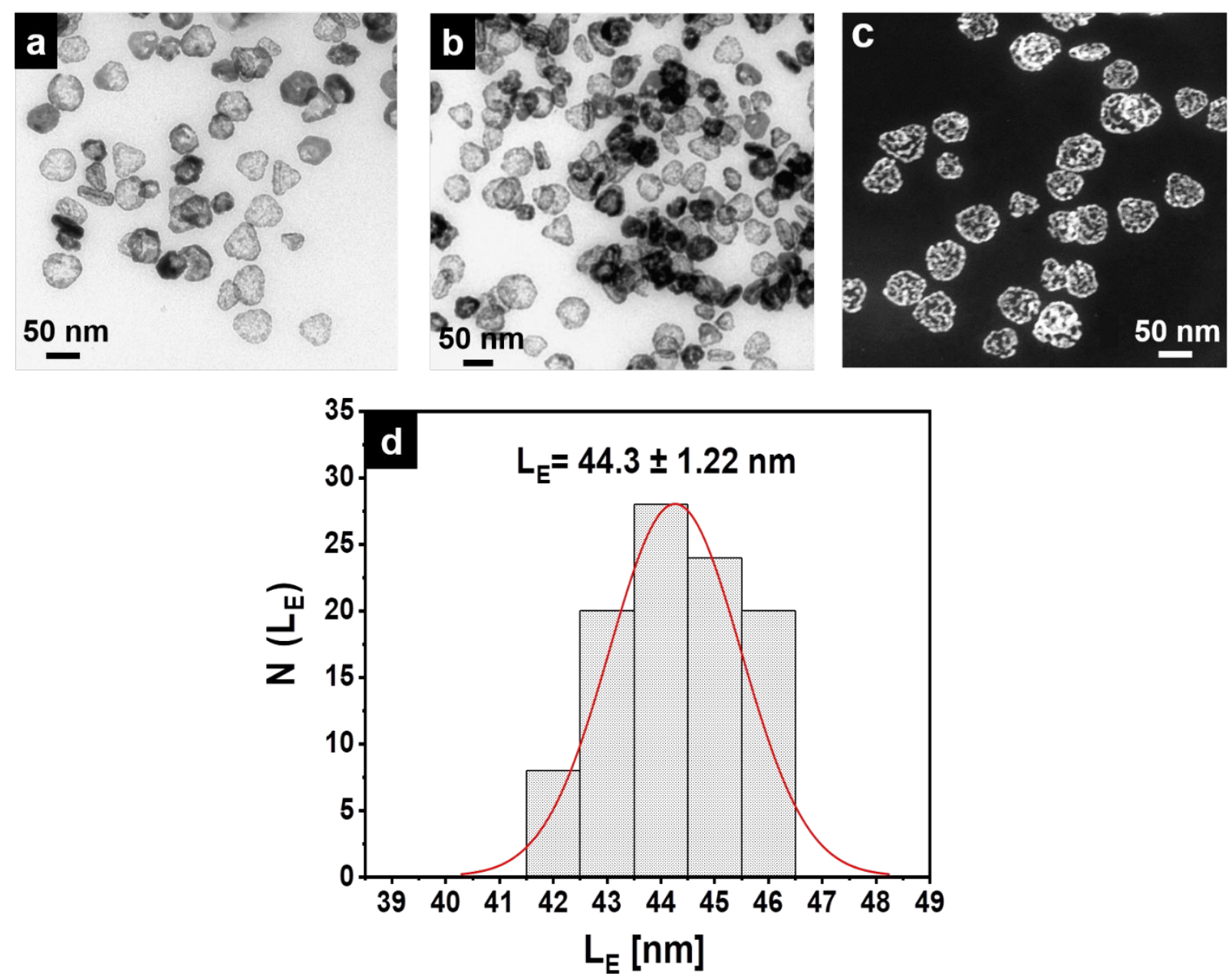

Figure S2. ( $a$ and b) TEM and (c) STEM images of AgPt NPS. (d) Size distribution histogram and size distribution Gaussian fitting of the edge length $\left(L_{E}\right)$ of AgPt NPs (mean edge length $L_{E}=$ $44.3 \pm 1.22 \mathrm{~nm}$, averaged from $100 \mathrm{NPs}$ ) 

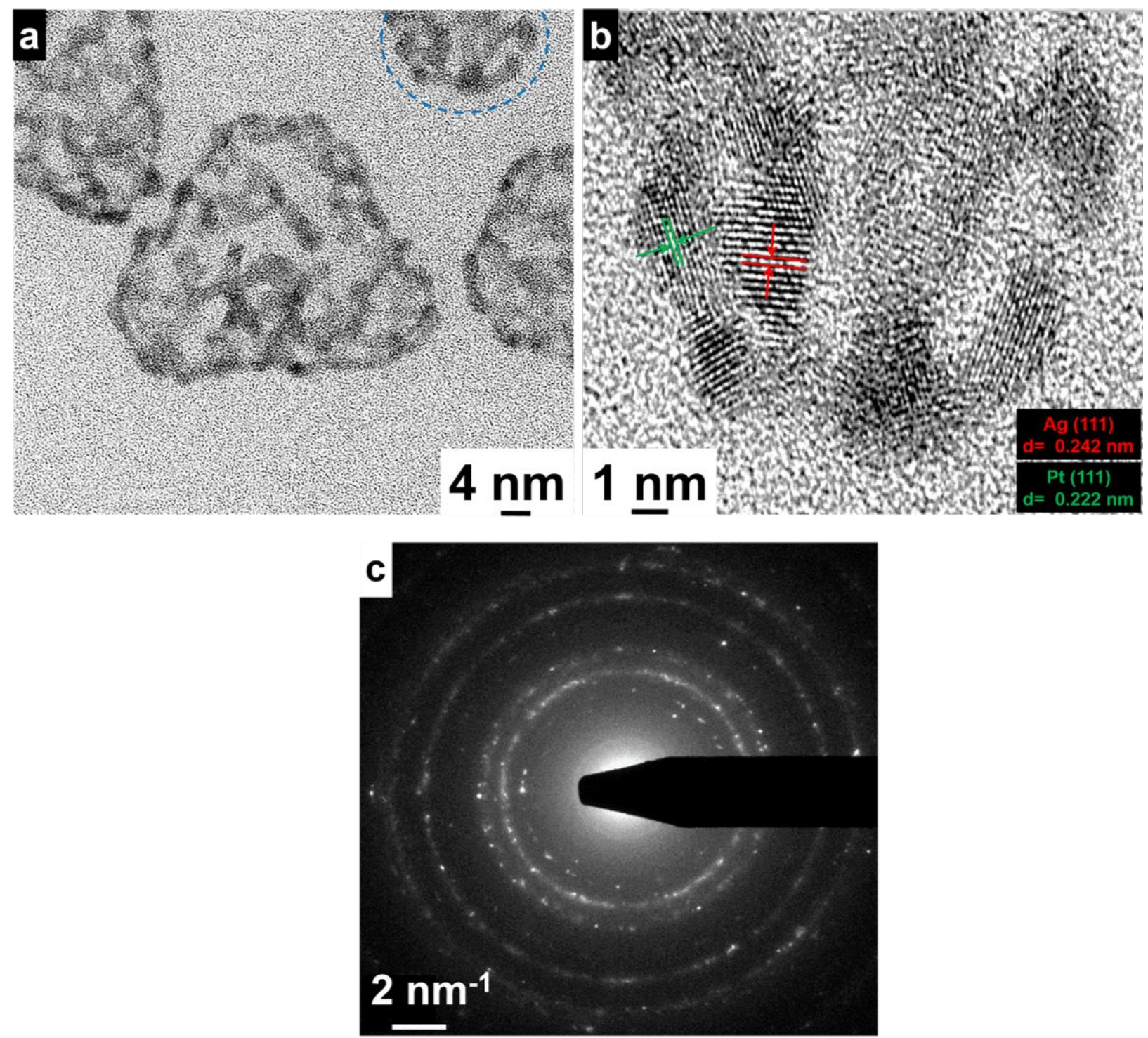

Figure S3. (a) High resolution transmission electron microscope (HRTEM) image of AgPt NPS and (b) HRTEM image of the outlined area in (a) showing the d-spacing for Ag and Pt in AgPt NPs. (c) SAED pattern of AgPt NPs showing a concentric ring pattern.

X-ray photoelectron spectroscopy (XPS) measurements were carried out ${ }^{3}$ using a high-resolution two-dimensional delay line 230 detector. A monochromatic Al K $\alpha$ X-ray source (photon energy $1486.6 \mathrm{eV}$; anode operating at $15 \mathrm{kV}$ ) was used to provide the incident radiation. XP spectra were recorded in fixed transmission mode. A pass energy of $40 \mathrm{eV}$ and $20 \mathrm{eV}$ were chosen for the survey and region scans, respectively, resulting in an overall energy resolution better than $0.4 \mathrm{eV}$. Charging effects were compensated by using a flood gun. The binding energies were calibrated based on the graphitic carbon $1 \mathrm{~s}$ peak at $285.0 \mathrm{eV}$. The base pressure was around $2 \times 10^{-10}$ bar. For sampling, all the NPs were concentrated and drop-casted on a $\mathrm{Cu}$ film, dried and then inserted 
in the instrument for the measurement. Casa XPS software was used to analyze and deconvolute all XP spectra ( $c f$. Figures S4 and S5).

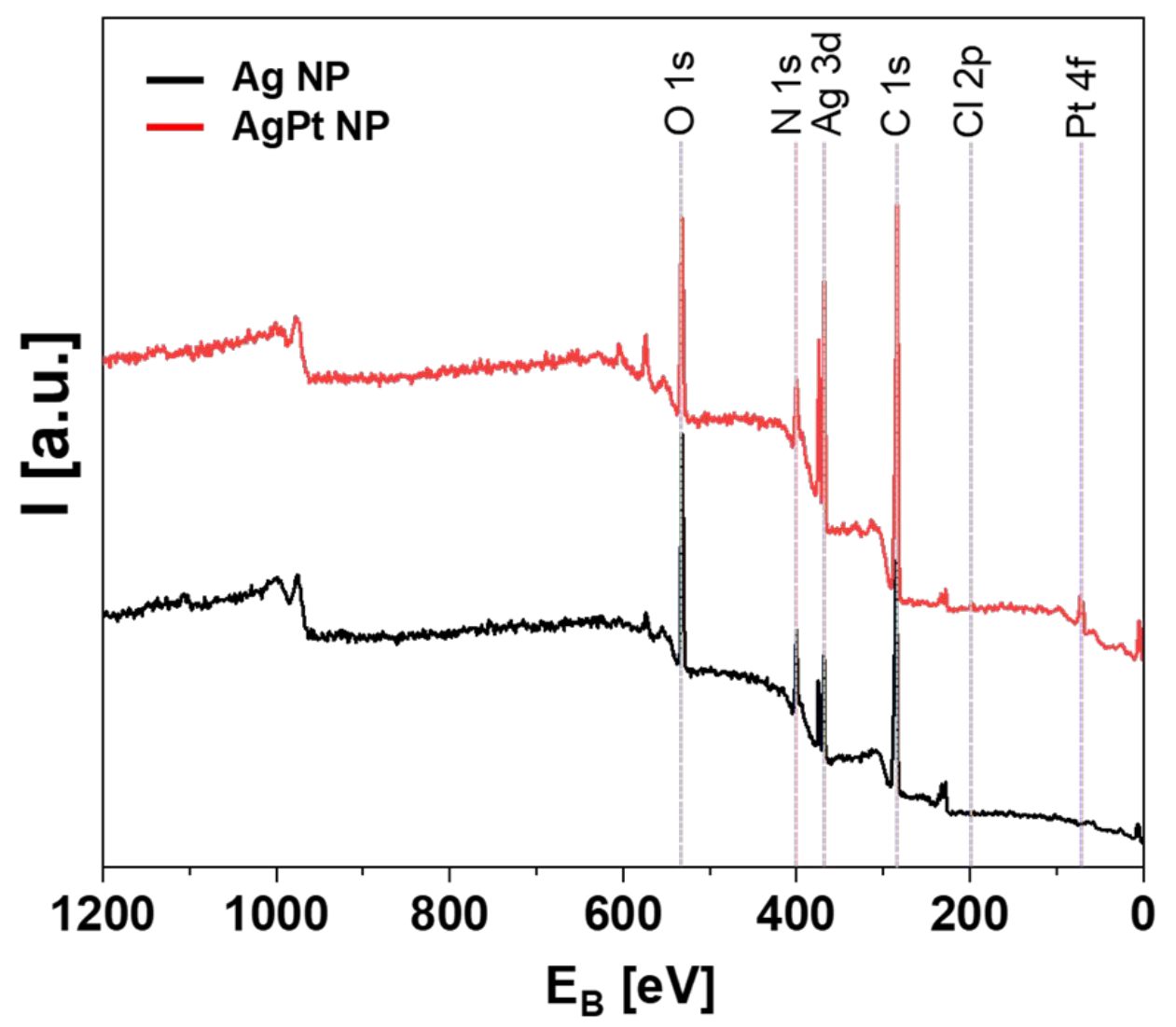

Figure S4. The XPS survey spectra of Ag NPs and AgPt NPs showing the existence of all the expected elements. Individual elements are assigned in the spectra. 

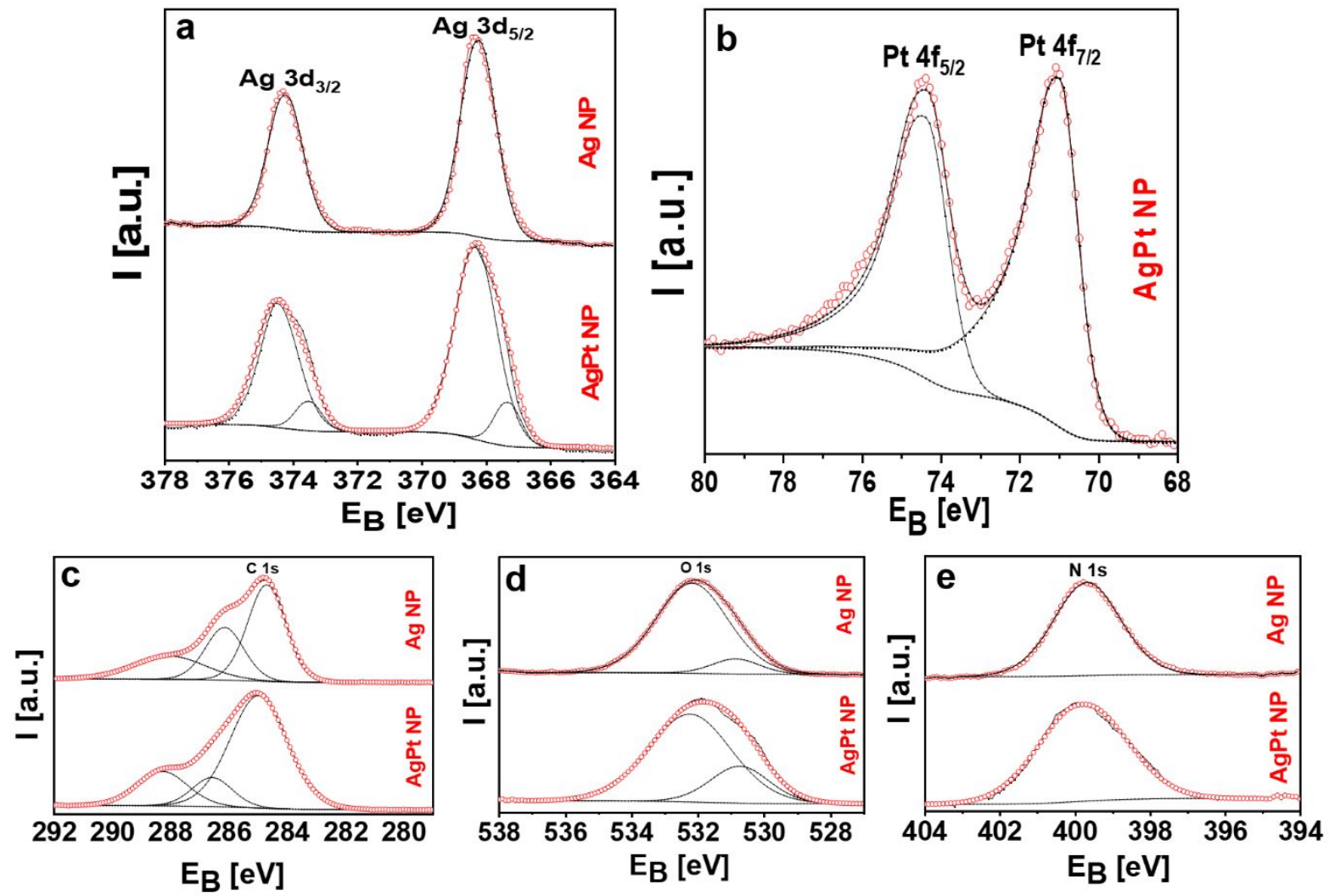

Figure S5. Deconvoluted XPS spectra of Ag3d (a), Pt4f (b), C1s (c), Ols (d) and N1s (e) respectively. All the spectral features have been fitted into the different chemical species expected. 


\section{Determination of NP concentration}

The concentration determination of complex-shaped NPs is in general complicated and often only can be estimated. ${ }^{4}$ One approach lies in estimating the volume per NP and from the density of the NP material, one can then calculate the number of atoms per NP. Elemental analysis of the number of atoms in a NP solution then allows for determining the number of NPs in the solution. ${ }^{5}$ It has to be pointed out that these numbers often are rough estimates, in particular for NPs with moderate size- and shape-distribution. First the surface area of the triangular prism $\left(A_{N P}\right)$ was calculated using the Heron's formula ${ }^{6}$ as follows:

$$
A_{N P}=\sqrt{S(S-a)(S-b)(S-c)}
$$

Where $a \simeq b \simeq c \simeq L_{E} \approx 45 \mathrm{~nm}$ (values roughly estimated from the TEM images (cf. Figure S2)) are the side lengths of the triangular prism and $S$ is its semiperimeter and calculated as follows:

$$
S=\frac{1}{2}(a+b+c)
$$

Then the volume of each triangular nanoprism $\left(V_{N \mathrm{P}}\right)$ was calculated by multiplying the surface area of each nanoprism by its height as follows.

$$
V_{N P}=\frac{1}{4} h \sqrt{2(a b)^{2}-a^{4}+2(a c)^{2}-b^{4}+2(b c)^{2}-c^{4}}
$$

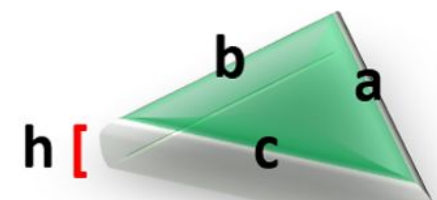

Where $h$ is the height of the prisms and is $\simeq 15 \mathrm{~nm}$. Therefore, the volume of one single NP $\left(V_{N P}\right)$ is $\approx 13152.76 \mathrm{~nm}^{3} \approx 1.3 \times 10^{-17} \mathrm{~cm}^{3}$. In turn, the mass of one single NP $\left(m_{N P}\right)$ can be determined by multiplying the volume of the NP by the material density $\left(\rho_{N P}\right)$ :

$$
m_{N P}=V_{N P} \times \rho_{N P}
$$

Giving that for the prism-shaped Ag NPs $\rho_{N P}=\rho_{A g}=10.5 \mathrm{~g} / \mathrm{cm}^{3}$, the weight of one single NP is $m_{N P} \approx 1.4 \cdot 10^{-16} \mathrm{~g}=1.4 \cdot 10^{-13} \mathrm{mg}$. 
The concentration of the NPs could be estimated by employing the data extracted from ICP-MS measurements where the Ag elemental concentration of purified NP solution was measured. In one representative experiment the final $\mathrm{Ag}$ concentration was $C_{A g}=84 \mathrm{mg} / \mathrm{L}$. The number concentration of NPs in the solution $\left(N_{N P}\right)$ can be calculated as follows:

$$
N_{N P}=C_{A g} / m_{N P}
$$

Where $C_{A g}$ is the elemental concentration of Ag.

In the representative example this led to $N_{N P} \approx 6 \times 10^{14} \mathrm{NPs} / \mathrm{L}$. The molar concentration of the NPs $\left(c_{N P}\right)$ was the calculated as follows:

$$
c_{N P}=N_{N P} / N_{A}
$$

Where $\mathrm{N}_{\mathrm{A}}$ is the Avogadro's number. Hence the stock concentration the NPs $\left(c_{N P}\right)$ in the example is $\approx 0.996 \mathrm{nM} \approx 1 \mathrm{nM}$. We want to emphasize again that this number is a rough estimate.

In case of AgPt NPs the NP concentration is first measured for the Ag NP templates as described above, and then the AgPt concentration after galvanic exchange is assumed to be the one of the original Ag NPs. 


\section{Colloidal stability of Ag NPs and AgPt NPs}

The colloidal stability of Ag NPs and AgPt NPs in sodium chloride $(\mathrm{NaCl})$ of different ionic strengths, phosphate-buffered saline (PBS), fetal bovine serum (FBS)-supplemented Dulbecco's Modified Eagle Medium (DMEM), FBS-free DMEM, FBS-supplemented Roswell Park Memorial Institute culture medium (RPMI), and FBS-free RPMI was studied using DLS. ${ }^{5} 100 \mu \mathrm{L}$ of NP solutions were taken in either $900 \mu \mathrm{L}$ of $\mathrm{NaCl}$ solutions of different ionic strengths $(25,50,100$, $150,300,600$ and $1250 \mathrm{mM}$ ) or in $900 \mu \mathrm{L}$ of PBS, FBS-supplemented DMEM, FBS-free DMEM, FBS-supplemented RPMI, and FBS-free RPMI, and their hydrodynamic diameters $\left(\mathrm{d}_{\mathrm{h}}\right)$ were measured directly after incubation $(0 \mathrm{~h})$ and $6 \mathrm{~h}, 12 \mathrm{~h}$, and $24 \mathrm{~h}$ post-incubation. The mean hydrodynamic diameters were determined from the intensity distribution curves of the hydrodynamics diameter $\left(\mathrm{d}_{\mathrm{h}}\right)$ measured by DLS. Data are presented in Figure S6.
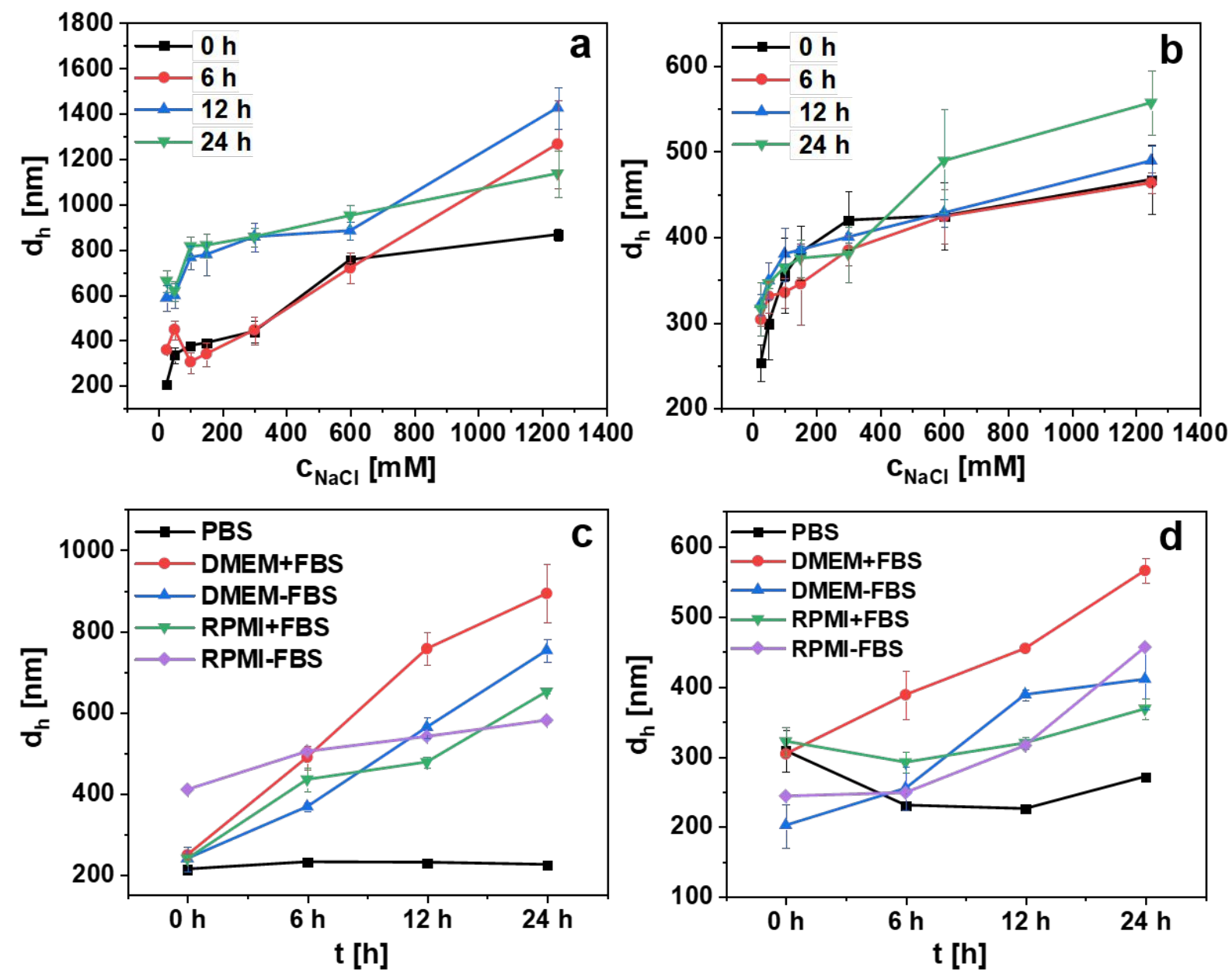

Figure S6. Colloidal stability of Ag NPs and AgPt NPs. Hydrodynamic diameters $\left(d_{h}\right)$ of Ag NPS and AgPt NPs in $\mathrm{NaCl}$ (a and b), respectively, and in different biological media (c and d), respectively. 


\section{Effect of pH on the synthesis of AgPt NPs}

The effect of changing the $\mathrm{pH}$ of the reaction medium on the formation of porous AgPt NPs was studied by recording the UV-vis absorption spectra of the obtained NPs. The $\mathrm{pH}$ of the reaction mixture (Ag NP sacrificial template solution has a $\mathrm{pH}$ of 3.8-4) was adjusted to $(2,6,8,10,12$ and 14) before the addition of Pt precursor and the UV-vis absorption spectra (Figure S7) of the corresponding solutions were collected. Further TEM images and EDS analyses of the reaction product at $\mathrm{pH} 4$ and $\mathrm{pH} 11$ are shown in Figure S13. The characteristic plasmon peaks of the $\mathrm{Ag}$ NPs were completely lost (pH 2) or partially lost ( $\mathrm{pH} 6$ and 8). Whereas, the characteristic UV-vis absorption spectra of the porous AgPt NPs were obtained only when the $\mathrm{pH}$ was 4 . The plasmon peaks of sacrificial Ag NPs did not change at higher $\mathrm{pH}(10,12$ and 14). These results show the importance of $\mathrm{pH}$ of the reaction on the synthesis product.

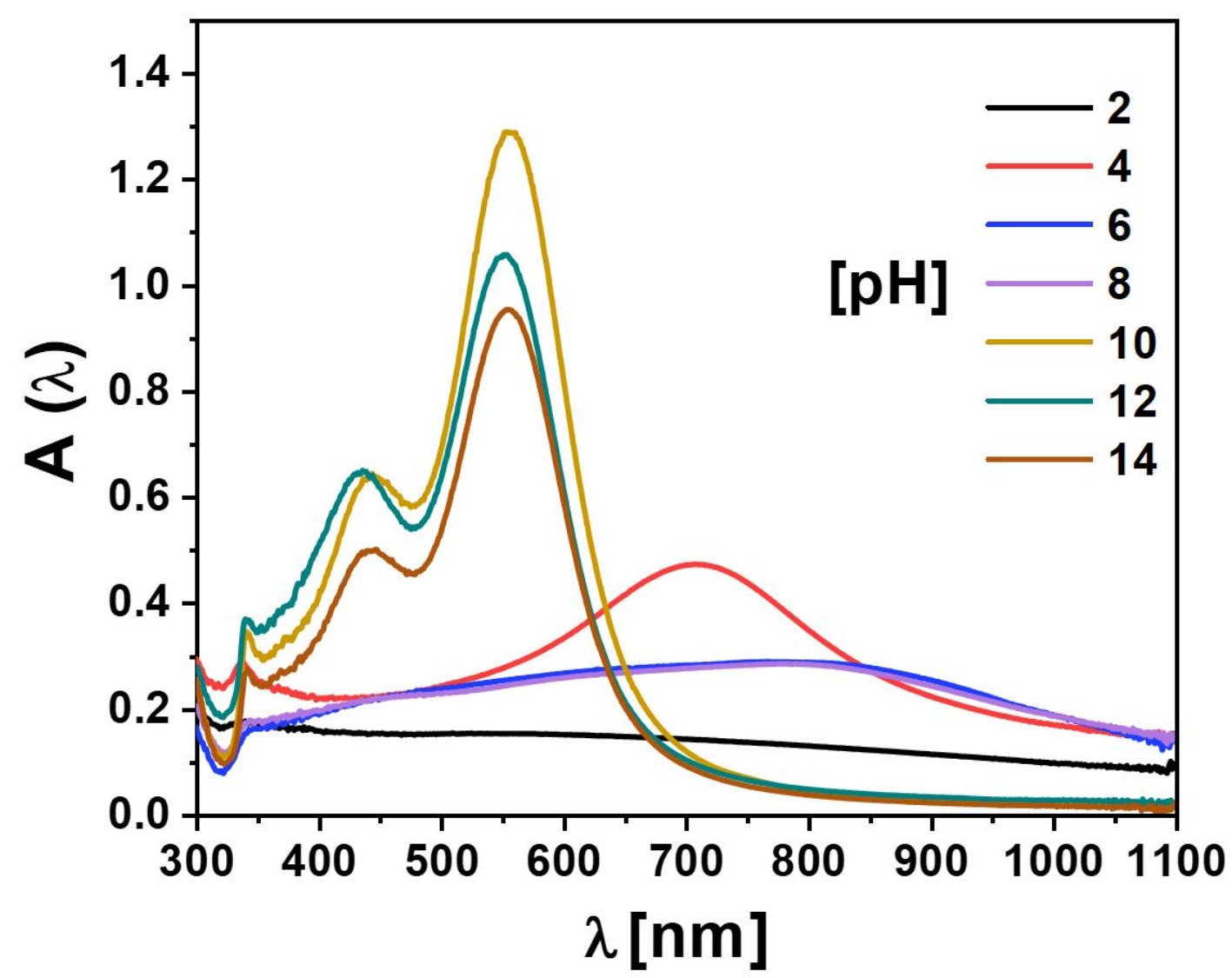

Figure S7. Effect of pH on the synthesis of AgPt NPs. UV-vis absorption spectra A( $\lambda)$ of different reaction products synthesized at wide range of $\mathrm{pH}(2-14)$. The $\mathrm{pH}$ was adjusted after the synthesis of the Ag NPs and before the addition of the Pt precursor. 


\section{Understanding the role of BSA in synthesis}

To confirm the key role of BSA in dictating the porosity of the AgPt NPs, the effect of replacing the surface-capping agent, BSA, with another ligand was studied.AgPt NPs@SC were synthesized by replacing BSA with sodium citrate (SC). All other chemicals used for the synthesis were kept the same.

\subsection{Synthesis of Ag NPs@SC and AgPt NPs@SC}

In a typical synthesis procedure, the growth of AgNPs@SC was initiated by mixing $50 \mu \mathrm{L}$ of $\mathrm{AgNO}_{3}(100 \mathrm{mM}), 50 \mu \mathrm{L}$ of sodium citrate $(100 \mathrm{mM})$, and $300 \mu \mathrm{L}$ of as-prepared $\mathrm{Ag}$ seed NP solution in a glass vial containing $10 \mathrm{~mL}$ of MilliQ water under vigorous stirring (1500-2000 rpm). Then, $60 \mu \mathrm{L}$ of ascorbic acid $(100 \mathrm{mM})$ was added subsequently, after which a series of color changes from faint-yellow to blue was observed. The formation of Ag NPs was completed in 2530 minutes and was confirmed by the appearance of the three characteristic absorption peaks of prism-shaped Ag NPs (Figure S8). The as-prepared NPs were centrifuged at $9000 \mathrm{rpm}$ for 15 minutes, washed two times with MilliQ water, and redispersed in MilliQ water for further experiments. Then, $50 \mu \mathrm{L}$ of platinum precursor $\left(\mathrm{H}_{2} \mathrm{PtCl}_{6}\right)$ was mixed with $10 \mathrm{~mL}$ of purified $\mathrm{Ag}$ NP@SC solution under vigorous stirring (1500 rpm) for 10-15 minutes at room temperature. The AgPt NPs@SC were purified by 2 consecutive cycles of centrifugation ( 9000 rpm, 15 minutes) and washing with MilliQ water. The final product was redispersed in MilliQ water for further work. The kinetics of AgPt NP@BSA as well as AgPt NP@SC formation was studied by collecting the UV-vis absorption spectra during the synthesis procedure. Aliquots from the reaction

mixtures were withdrawn and their UV-vis absorption spectra at 2 minute intervals were collected until no further change in the spectra was noticed. The data of the kinetics of AgPt NPs@BSA and AgPt NPs@SC are shown in Figure S9.

\subsection{Characterization of Ag NPs@SC and AgPt NPs@SC}

UV-vis absorption spectra of Ag NP@BSA, Ag NP@SC, AgPt NP@BSA, and AgPt NP@SC as well as DLS measurements of hydrodynamic diameter $\left(\mathrm{d}_{\mathrm{h}}\right)$ and TEM images of AgPt NP@SC and AgPt NP@BSA were collected to elucidate the role of surface ligand in dictating the morphology of the NPs. 

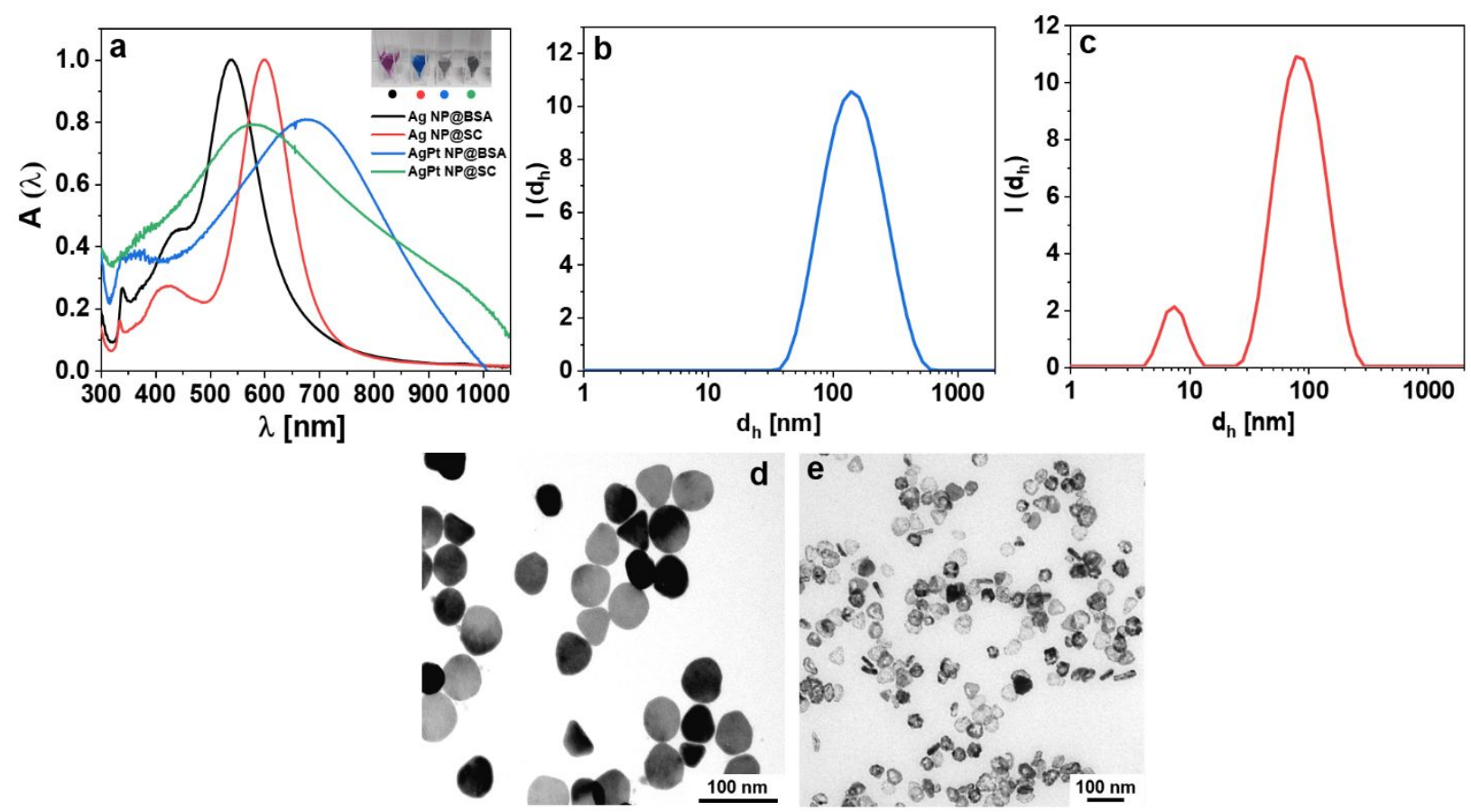

Figure S8. Role of BSA in directing the shape towards porous prism-shaped AgPt NPs. (a) UVvis absorption spectra A( $\lambda)$ of Ag NP@BSA,AgNP@SC,AgPt NPs@BSA, and AgPt NPs@SC and their corresponding photographs in the inset. ( $b$ and $c$ ) Hydrodynamic diameter $d_{h}$ of AgPt NPs@SC and AgPt NPs@BSA as recorded in water in the form of the intensity distribution I(dh), respectively. (d and e) TEM images of AgPt NPs@SC and AgPt NPs@BSA, respectively.
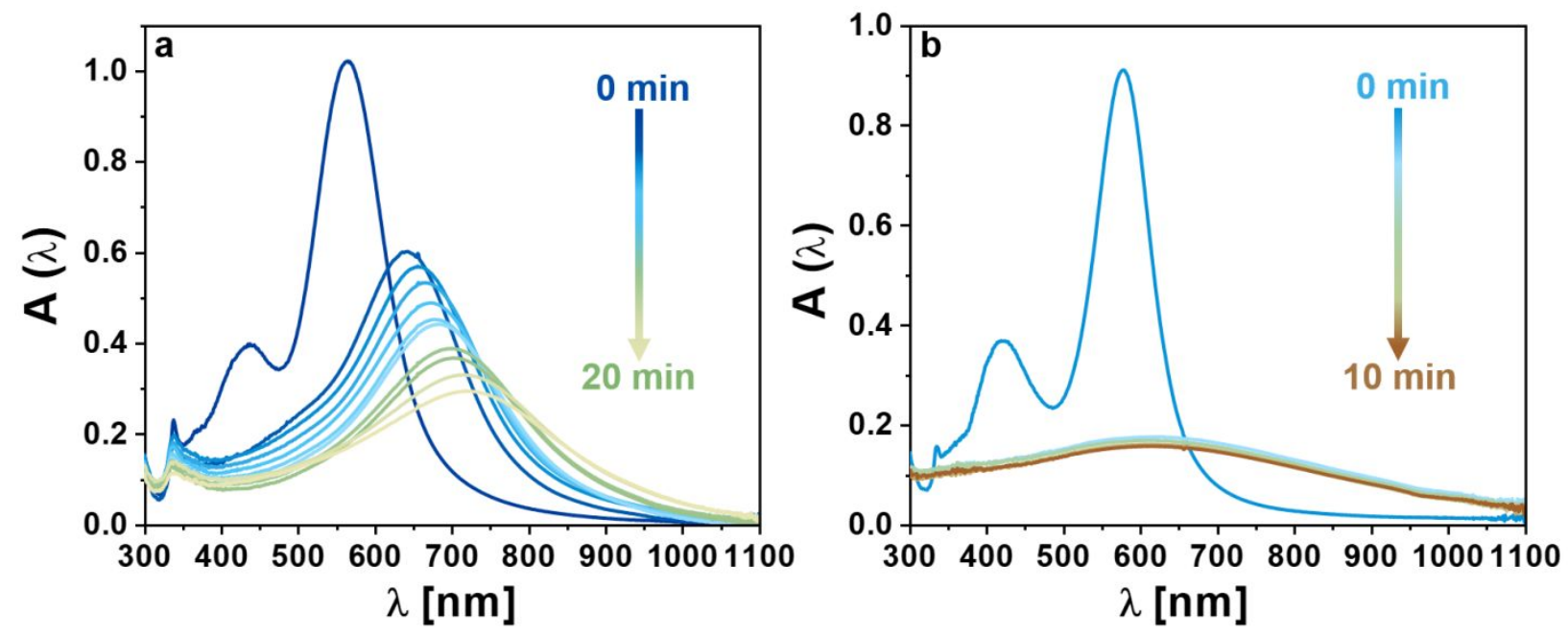

Figure S9. Kinetics of AgPt NP formation with different surface ligands. UV-vis absorption spectraA( $\lambda$ ) recorded at different time points during the synthesis of (a)AgPt NPs@BSA and (b) AgPtNPs@SC. 


\section{Effect of Pt precursor $\left(\mathrm{H}_{2} \mathrm{PtCl}_{6}\right)$ concentration on the synthesis}

The effect of changing the concentration of Pt precursor on the optical properties, morphology, as well as the elemental composition of the AgPt NPs was investigated. After the synthesis of the sacrificial Ag NP template, different amounts (50, 100, 200 and $500 \mu \mathrm{L})$ and $(10,20,50$ and 100 $\mu \mathrm{L}$ ) of $2 \mathrm{mM}$ and $25 \mathrm{mM} \mathrm{Pt}$ precursor, respectively, were injected to the reaction mixture. Then, the UV-vis absorption spectra were collected after 20 minutes. The absorption spectra did not show significant change with the relatively low Pt precursor concentration $(2 \mathrm{mM})$, whereas using relatively higher Pt precursor $(25 \mathrm{mM})$ was accompanied by a significant red-shift and damping of the plasmonic peaks and loss of some plasmonic peaks features of the Ag NPs (Figures S10 a and b). Systematic concentration-dependent experiments were carried out to understand the effect of different Pt concentrations (by changing the molar ratios of Ag and Pt precursor) on synthesis of the NPs. The corresponding UV-vis absorption spectra are presented in Figure S10c.

Furthermore, the TEM images and EDS elemental composition of AgPt NPs formed using different Ag:Pt molar ratio were collected. In these experiments a fixed amount $(50 \mu \mathrm{L})$ of varied concentrations of Pt precursor ( $5 \mathrm{mM}, 10 \mathrm{mM}, 25 \mathrm{mM}, 50 \mathrm{mM}$ and $100 \mathrm{mM})$ was injected into equal amounts of Ag NP sacrificial templates, which corresponds to Ag:Pt molar ratios of (1:0.05, 1:0.1, 1:0.25, 1:0.5 and 1:1), respectively. The AgPt NPs which were synthesized with 1:0.05 and 1:0.1 Ag:Pt showed the characteristic morphology of solid prism-shaped NPs with atomic percentage of $\mathrm{Ag}$ (94.1\% and 89.8\%), respectively, and Pt content of (5.9\% and and $10.2 \%$ ), respectively (Figures S11 a and b). At Ag:Pt molar ratio of 1:0.25, the Pt concentration is high enough to induce oxidative dissolution of $\mathrm{Ag}$ atoms of the $\mathrm{Ag}$ NPs and subsequent $\mathrm{Ag}^{+}$ion release in the reaction medium takes place, which leads to the formation of small cavities in the Ag NPs and simultaneous Pt atom deposition and initiation of the alloying process. The porous AgPt NPs at this unique Ag:Pt molar ratio exhibit bimetallic NPs with $48.5 \% \mathrm{Ag}$ and $51.5 \% \mathrm{Pt}$ atomic percentage (Figure $1 \mathrm{~b}$-f). A further increase of the Ag:Pt molar ratio to 1:0.5 and 1:1 was accompanied by de-alloying and surface reconstruction processes which lead to the rupture of the $\mathrm{AgPt}$ nanostructure (Figures S11 $\mathrm{c}$ and d). The same trend was observed in many alloy NP systems. $^{7}$ 

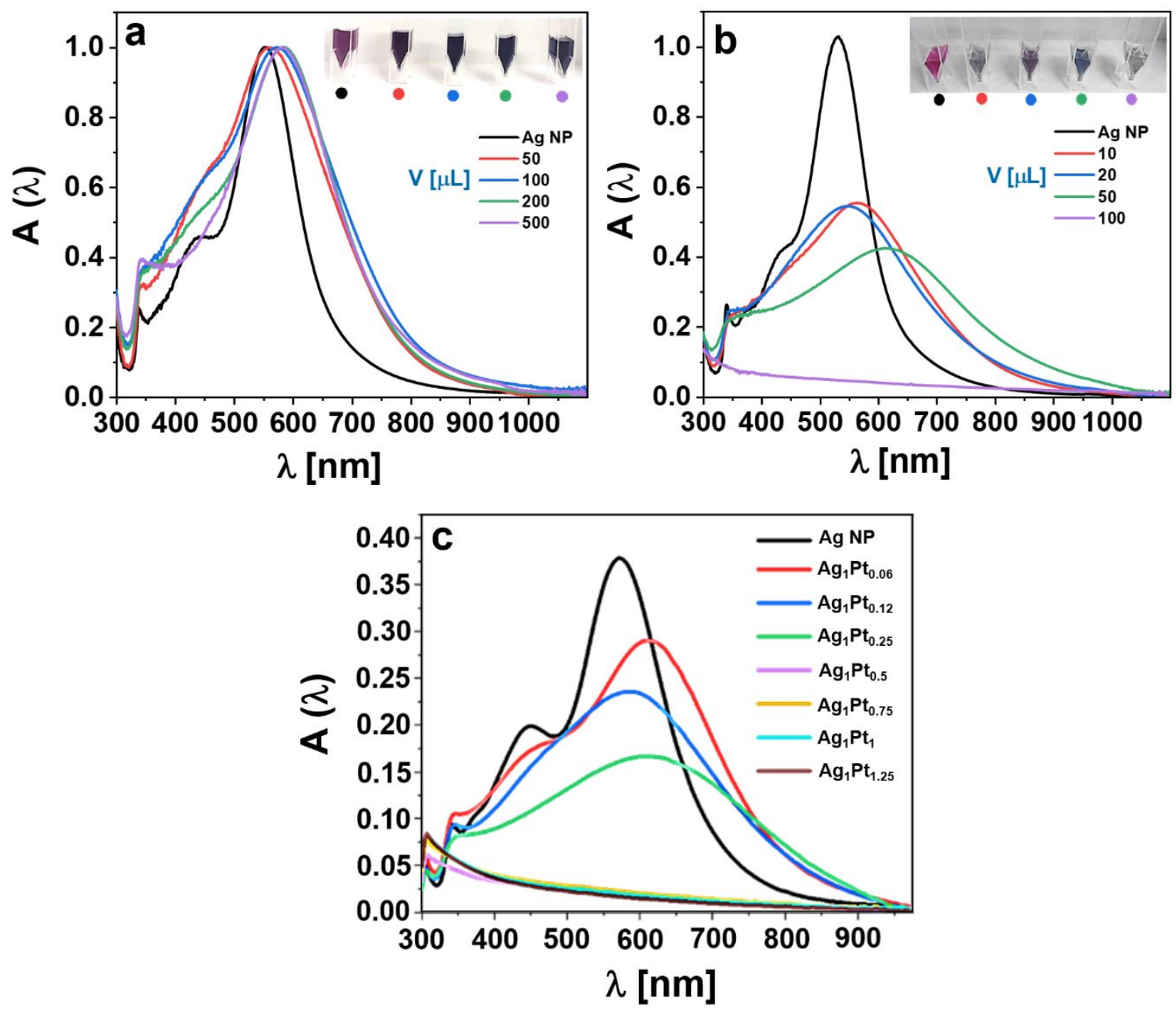

Figure S10. Effect of using different concentrations of Pt precursor $\left(\mathrm{H}_{2} \mathrm{PtCl}_{6}\right)$ on the synthesis product. UV-vis absorption spectra $A(\lambda)$ of the NPs prepared using different amounts (V) of (a) 2 mM and (b) $25 \mathrm{mM}$ Pt precursor (Insets are the photographs of the AgPt NPs prepared using various amount of $2 \mathrm{mM}$ and $25 \mathrm{mM}$ Pt precursors). (c) UV-vis absorption spectra $A(\lambda)$ of the AgPt NPs prepared using different molar ratios of Ag and Pt precursor. 

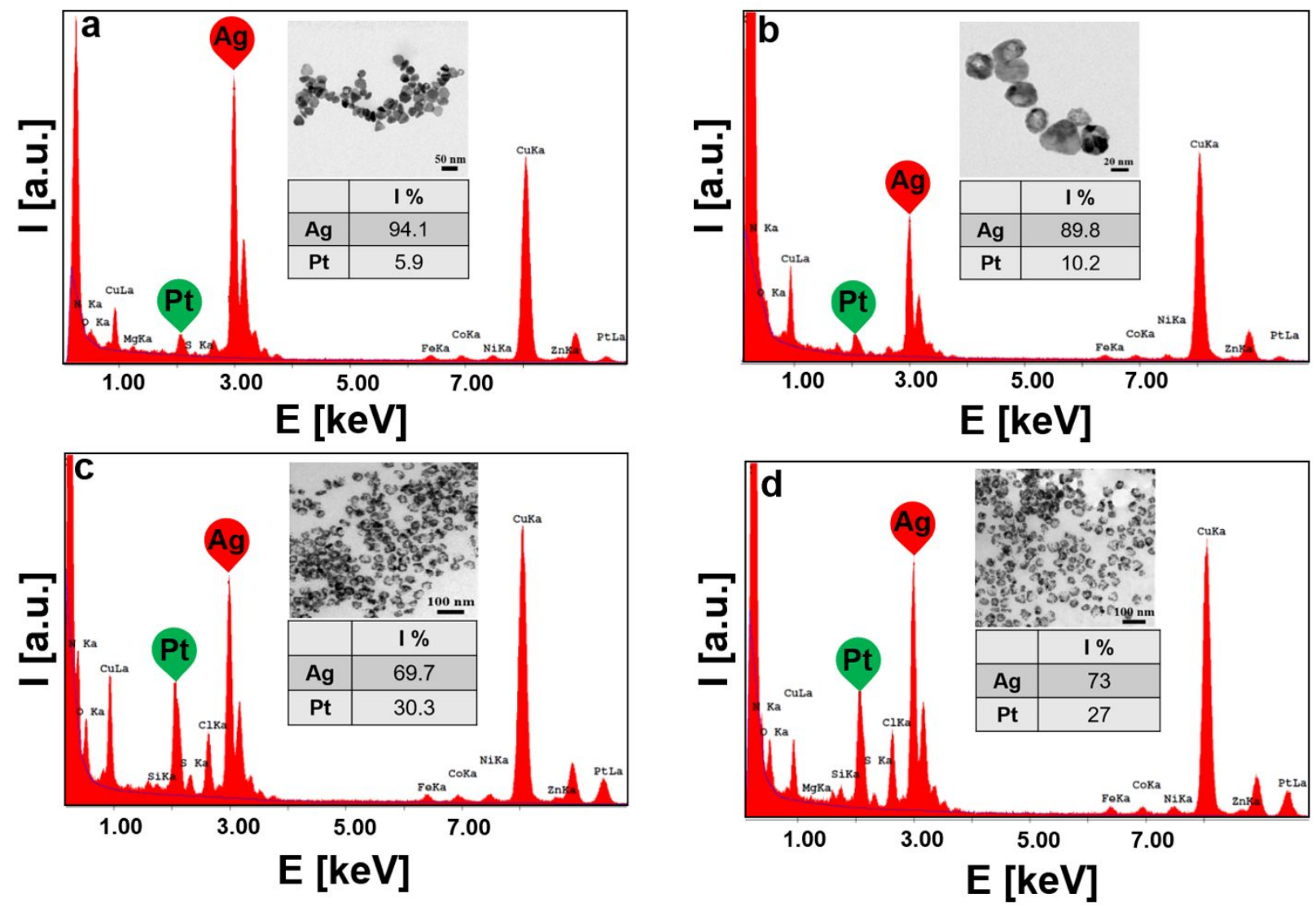

Figure S11. Effect of changing the Ag:Pt molar ratio on the NP shape and composition. (a-d) EDS spectra of AgPt NPs prepared using 5, 10, 50 and $100 \mathrm{mM} \mathrm{Pt}$ precursor, respectively. Insets are the corresponding TEM images and tables summarizing the atomic \% of Ag and Pt obtained from the EDS spectra. The results obtained during the synthesis of AgPt NPs using 25 mM Pt precursor are shown in Figure 1(b-f). 


\section{Other bimetallic AgM NPs using the same synthesis protocol}

To demonstrate the versatility of our synthesis approach, the ability of BSA to direct the synthesis of anisotropic AgM NPs other than AgPt NPs was investigated. The AgM (M=Pd and Au) NPs were synthesize following the same protocol used for the synthesis of AgPt NPs (cf. $\S 3$ ). UV-vis absorption spectra, TEM images as well as elemental composition of the reaction products measured at different $\mathrm{pH}$ (4 and 11) were collected. The data obtained from the absorption spectra, EDS analyses suggest maximum incorporation of $\mathrm{Pt}(51.5 \%)$ and $\mathrm{Pd}(24.2 \%)$ in the NPs at $\mathrm{pH} 4$, whereas maximum incorporation of $\mathrm{Au}$ was exhibited at $\mathrm{pH} 11$ (19\%) (Figures S12-S15). Out of the three metals used ( $\mathrm{Pt}, \mathrm{Pd}$ and $\mathrm{Au}$ ), Pt and Au were able to form porous NPs at $\mathrm{pH} 4$ and $\mathrm{pH} 11$, respectively, as could be shown from TEM images (Figures S13a and S15c). This could also be shown from the UV-vis absorption spectra (Figure S12), which show disappearance of the shoulder peak attributed to the in-plane quadrupole resonance and the broadening of the plasmon peak attributed to the in-plane dipole resonance.
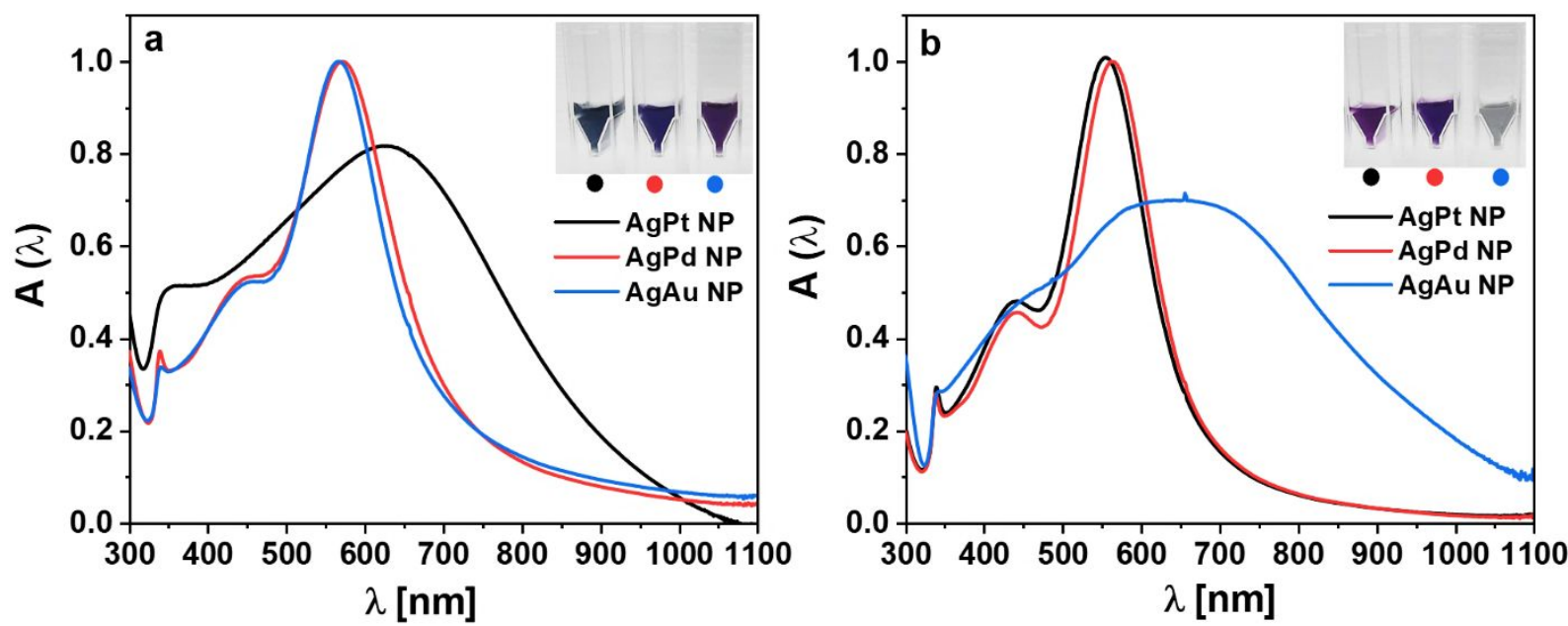

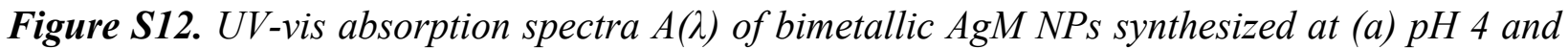
(b) $\mathrm{pH} \mathrm{11,} \mathrm{respectively} \mathrm{(Insets} \mathrm{are} \mathrm{the} \mathrm{photographs} \mathrm{of} \mathrm{the} \mathrm{AgM} \mathrm{NPs} \mathrm{prepared} \mathrm{at} \mathrm{pH} 4$ and 11, respectively). 

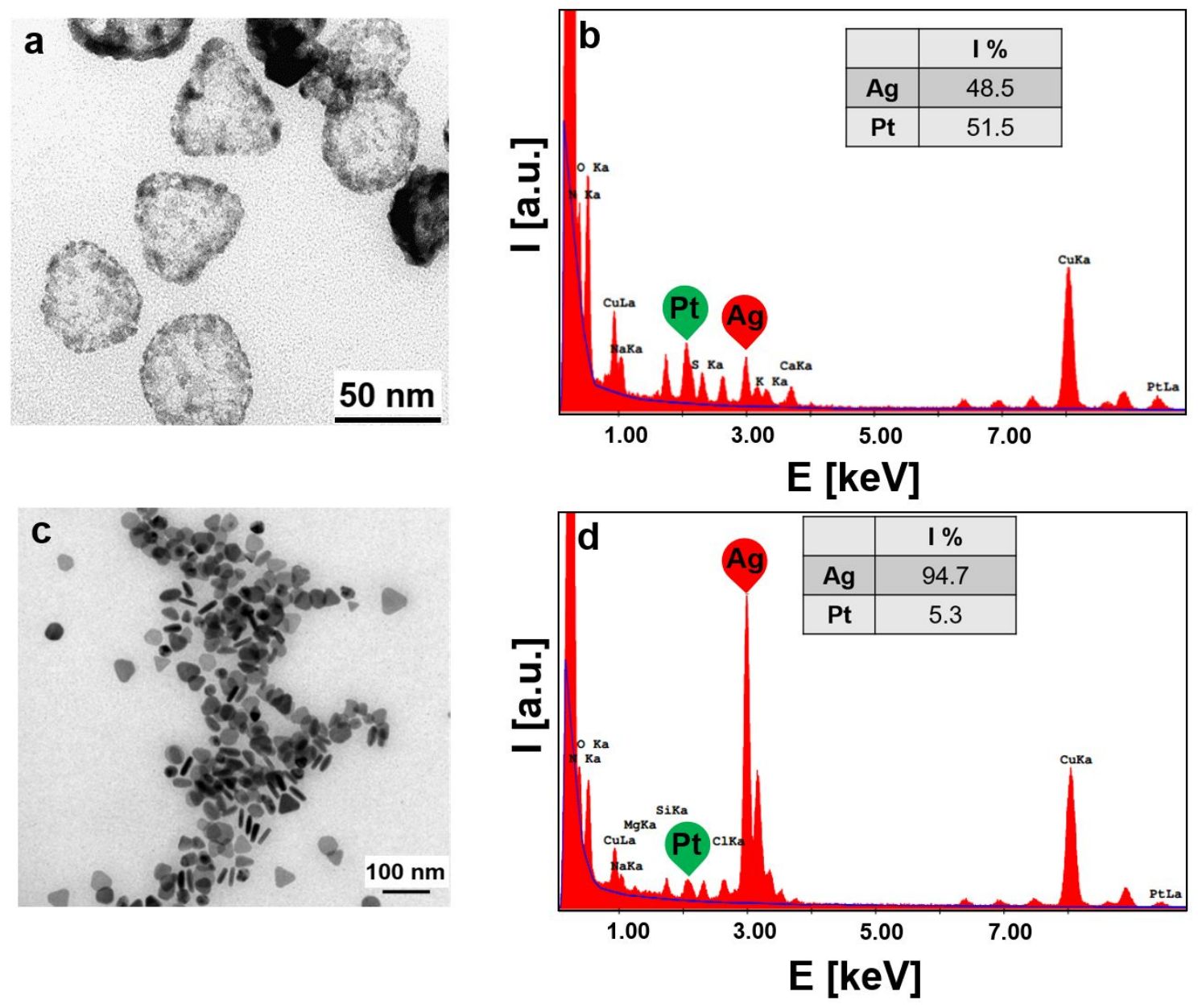

Figure S13. TEM images and EDS spectra of AgPt NPs prepared at (a-b) $p H 4$ and (c-d) $p H 11$, respectively. Insets in (b) and (d) are the atomic \% of Ag and Pt in the AgPt NPs prepared at the corresponding $\mathrm{pH}$. The data represented in (b) is the same as shown in Figure $1 f$. 

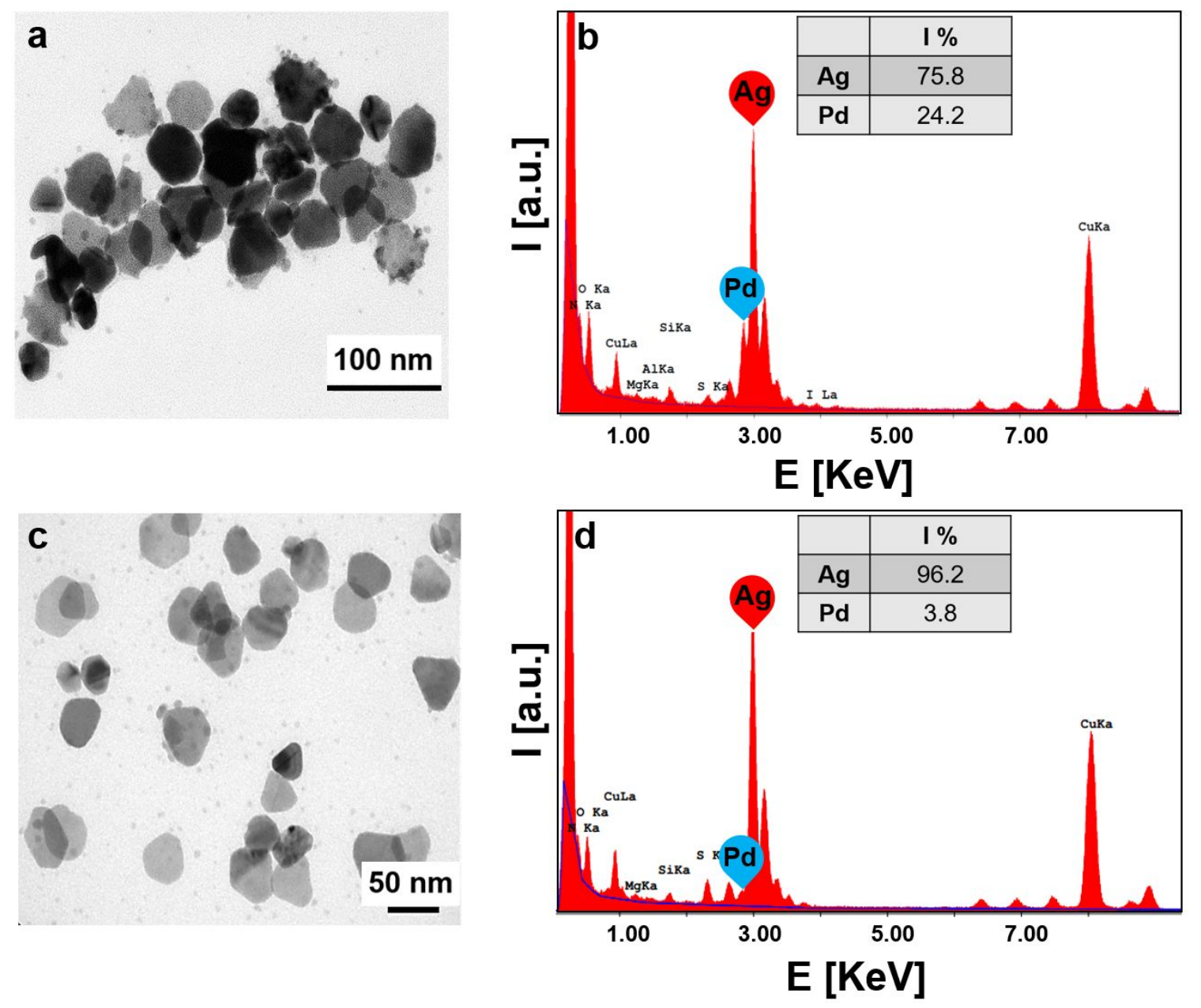

Figure S14. TEM images and EDS spectra of AgPd NPs prepared at (a-b) $p H 4$ and (c-d) $p H 11$, respectively. Insets in (b) and (d) are the atomic \% of Ag and Pd in the AgPd NPs prepared at the corresponding $\mathrm{pH}$. 

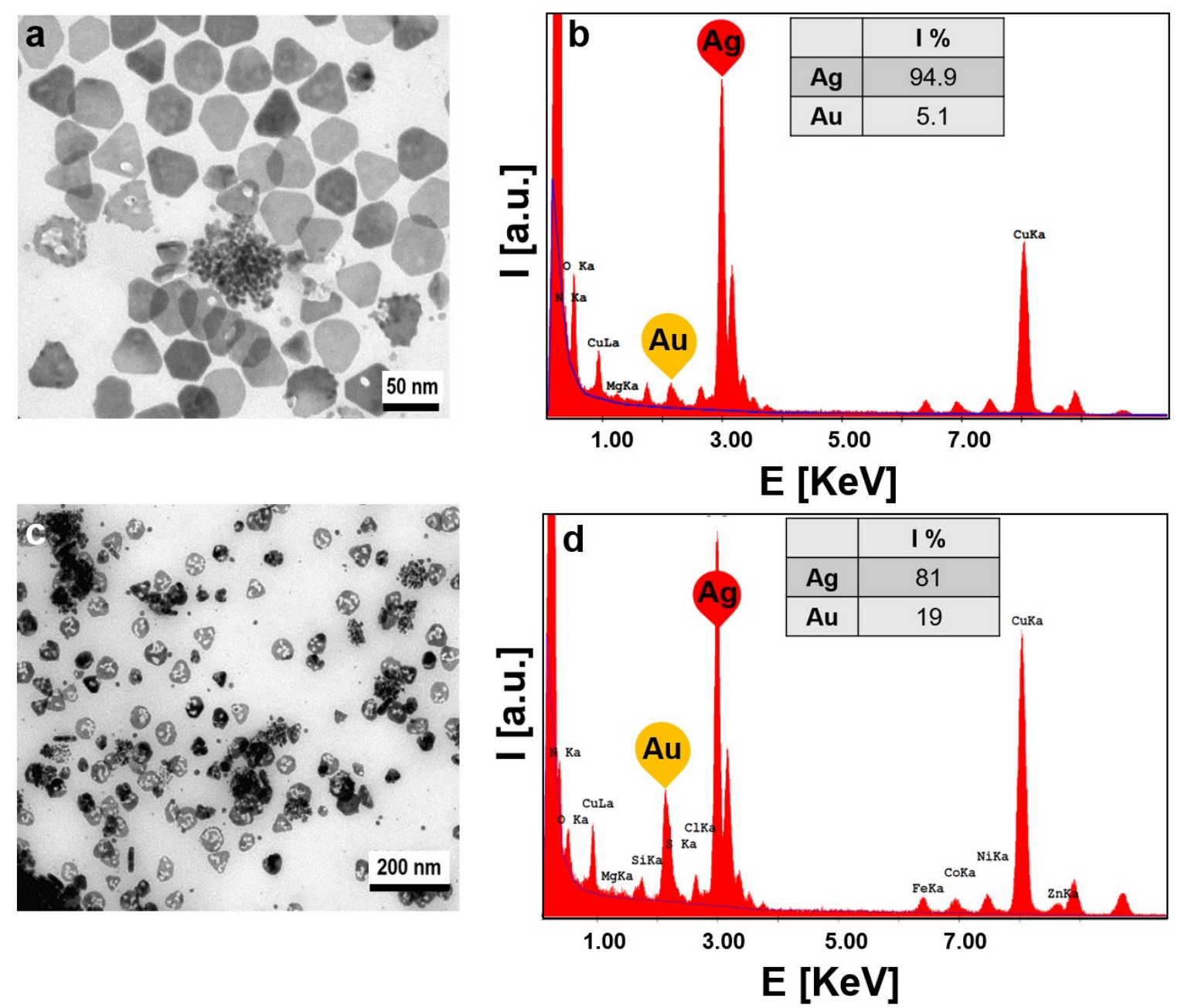

Figure S15. TEM images and EDS spectra of AgAu NPs prepared at (a-b) $p H 4$ and (c-d) $p H 11$, respectively. Insets in (b) and (d) are the atomic \% of Ag and Au in the AgAu NPs prepared at the corresponding $\mathrm{pH}$. 


\section{Structural changes of BSA during the synthesis of Ag NPs and AgPt NPs}

\subsection{Circular dichroism spectroscopy measurements}

To reveal the role of protein in directing the shape of synthesized NPs and to obtain insights into the evolution of secondary structures of BSA during the synthesis of our AgPt NPs, the conformational modifications, folding and binding properties of BSA molecules were evaluated using circular dichroism (CD). CD spectra of native BSA in neutral $\mathrm{pH}(6.5, \mathrm{~N}$-form) and acidic $\mathrm{pH}$ ( $\mathrm{pH}$ 4, E-form), Ag NP and AgPt NP solutions were recorded on a Chirascan ${ }^{\mathrm{TM}}$ Circular Dichroism Spectrometer (Applied Photophysics Ltd., UK) from 190 to $260 \mathrm{~nm}$ at $25^{\circ} \mathrm{C}$ using $1 \mathrm{~nm}$ bandwidth and $2 \mathrm{~mm}$ path length quartz cuvettes. The CD spectra were recorded, averaged over three scans and the data were then introduced to a freely available web server "http://bestsel.elte.hu" that uses the Beta Structure Selection method (BeStSel), which implements an algorithm used for the secondary structure determination and fold recognition of a protein and takes into consideration the twist of $\beta$-structures. This method can effectively distinguish parallel and antiparallel $\beta$-sheets and offers an improved estimation of the secondary structure for a broad range of proteins. ${ }^{8}$

The distribution of secondary structure elements of BSA within the N- and E-forms of BSA, Ag NPs and AgPt NPs as estimated from the CD spectra (Figure 1g) using the online protein CD spectra deconvolution web server "http://bestsel.elte.hu" is shown in Figure S16 (a-d) and the relative contents of secondary structure elements of BSA obtained thereof are summarized in Figure S16e. This evaluations is similar to the one in a previous report. ${ }^{9}$ 


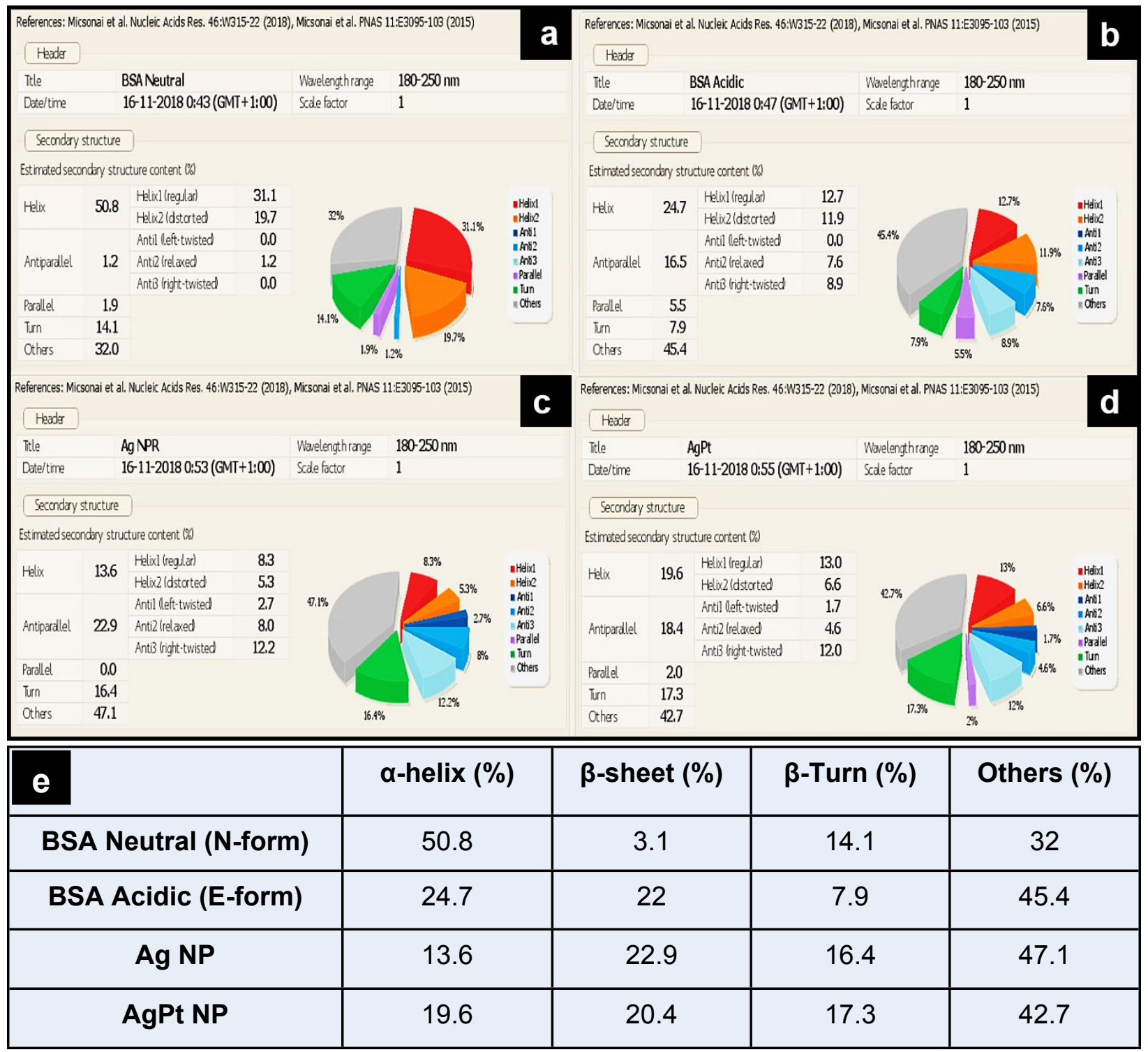

Figure S16. Distribution of secondary structure elements of BSA within the (a) $N$ - and (b) Eforms of BSA, (c) Ag NPs and (d) AgPt NPs as estimated from the CD spectra using online web server for protein CD spectra deconvolution "http://bestsel.elte.hu”. (e) Table summarizing the relative content (\%) of secondary structure elements of BSA as obtained from (a-d).

\subsection{Fluorescence spectroscopy measurements}

To further investigate the nature of interaction between BSA and Ag as a determining step during the synthesis of the sacrificial template, fluorescence experiments were carried out. The intrinsic fluorescence behavior of BSA originates from two tryptophan residues (Trp-134 in domain I of the BSA and Trp-213 in domain II). It had been shown that the fluorescence intensity and the position of the peak emission wavelength (emission maxima) depend on the degree of exposure of 
the Trp-side chains to polar solvents ${ }^{10-11}$ as well as the properties of the environment around the tryptophyl residues ${ }^{12}$, respectively. The conformational alternation of BSA during the synthesis of Ag NPs could be probed by the selective excitation of Trp-residues at $295 \mathrm{~nm} .{ }^{9}$ Changes in fluorescence emission intensity as well as the emission maxima upon excitation at $295 \mathrm{~nm}$ were recorded. Our results show that the fluorescence intensity of the E-form of BSA ( $344 \mathrm{~nm}, 3.8 \times 10^{6}$ CPS) was lower than that of the $\mathrm{N}$-form $\left(352 \mathrm{~nm}, 7.8 \times 10^{6} \mathrm{CPS}\right)$ in addition to a slight blue shift in the emission peak maximum from $352 \mathrm{~nm}$ (N-form) to $344 \mathrm{~nm}$ (E-form) (Figure S17). We had shown that the E-form of BSA exhibits low degree of helicity than that of the $\mathrm{N}$-form, which is in agreement with previous work which correlated the diminished fluorescence intensity with the loss of $\alpha$-helical structures of BSA. ${ }^{13} \mathrm{Ag}$ NPs and in turn AgPt NPs show a decrease in the fluorescence intensity of the pristine BSA $\left(2.3 \times 10^{5}\right.$ and $1.8 \times 10^{5} \mathrm{CPS}$, respectively) with a red shift of the emission maxima ( $387 \mathrm{~nm}$ and $361 \mathrm{~nm}$, respectively) (Figure S17). Such decrease in the intensity is explained in part by the decrease in the $\alpha$-helical structures in both Ag NPs and AgPt NPs in comparison to the N- and E-forms of BSA, as well as due to the increased probability of Trpresidues being present in close proximity to $\mathrm{Ag}$ atoms. The red shift of the emission maxima indicates a conformational change in the BSA molecules, which makes the Trp-residues more accessible for polar environment. Taken these results together along with our findings from the CD measurements, we hypothesize that the growth of the sacrificial Ag NPs proceeds with i) a conformational change in the BSA molecules and a subsequent protein unfolding due to the low $\mathrm{pH}$ environment, and ii) a simultaneous preliminary $\mathrm{Ag}^{+}$reduction by $\mathrm{AA}$ and formation of $\mathrm{AgNPs}$. BSA contains amino acid residues that provide both recognition sites, to bind strongly and specifically to Ag nanoclusters, and reduction sites as well, to further reduce more Ag ions. BSA more likely brings about the face-selective growth by a strong preferential binding to the $\{111\}$ facets of Ag, in addition to its mild reducing capability that comes from the hydroxyl groups of the threonine and serine units revealed upon BSA unfolding, which slows down the reduction rate and hence directs the growth towards the triangular shape. This unique binding leads to a lower surface energy of the $\{111\}$ faces of the crystal lattice and blocks the overgrowth of $\mathrm{Ag}^{0}$ onto the $\{111\}$ and allows therefore the crystal growth by permitting the accumulation of $\mathrm{Ag}^{0}$ onto other facets rather than the $\{111\}$ such as the $\{100\}$ facets, which in turn facilitates the formation of prism-shaped NPs. 

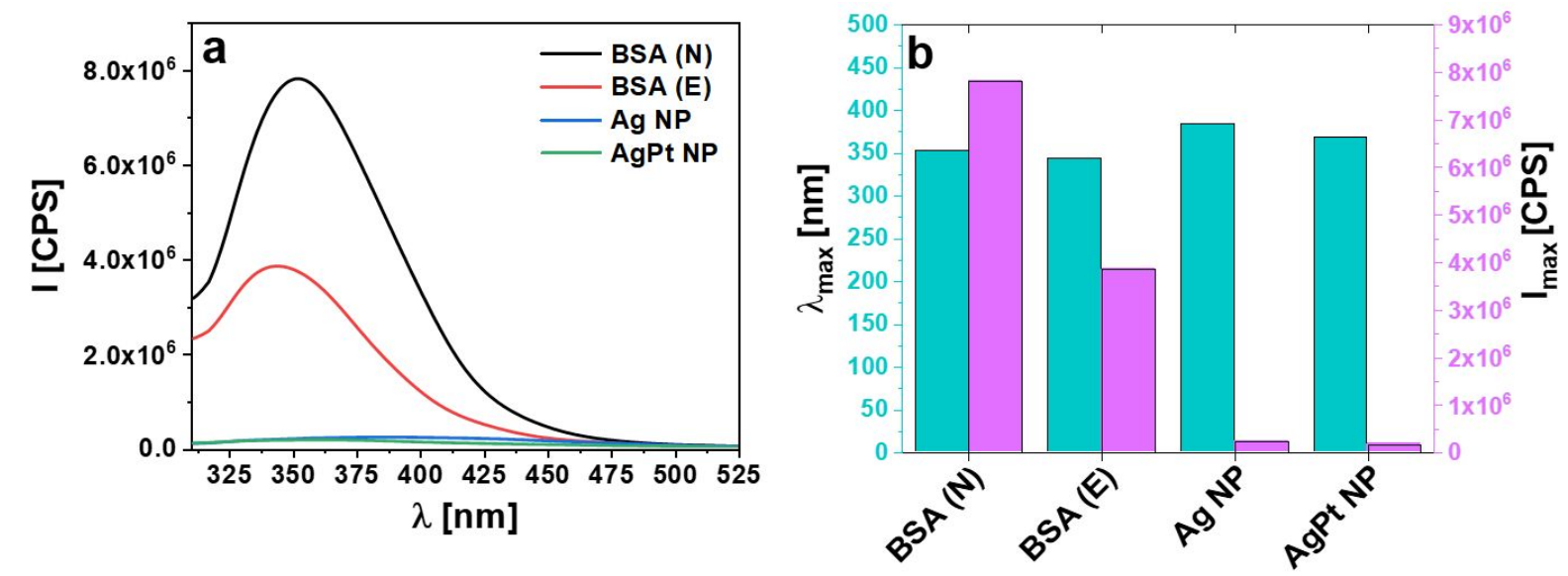

Figure S17. The structural changes of BSA during the synthesis of Ag NPs and AgPt NPs. (a) Fluorescence spectra $\left(\lambda_{e x}=280 \mathrm{~nm}\right)$ of the $N$ - and E-forms of BSA, Ag NPs, and AgPt NPs and (b) their peak emission intensity ( $I_{\max }$, purple columns) and peak emission wavelength $\left(\lambda_{\max }\right.$, turquoise columns). 


\section{Peroxidase-like activity of AgPt NPs}

The peroxidase-like activity of AgPt NPs was investigated through the catalytic oxidation of the nearly colorless peroxidase substrate ortho-phenylenediamine (OPD) in the presence of $\mathrm{H}_{2} \mathrm{O}_{2}$ to produce a yellow colored 2,3-diaminophenazine (DAP). A typical experiment was performed at $37^{\circ} \mathrm{C}$ by mixing $25 \mu \mathrm{L}$ of AgPt NPs (Final concentration of NPs $\left(c_{N P}\right)=0.0332 \mathrm{nM}$, details of the determination of the NP concentration are mentioned in $\S 5$ ) in a reaction volume of $3 \mathrm{~mL}$ sodium acetate buffer $(\mathrm{pH}=4)$ with the substrate OPD $(0.32 \mathrm{mM}$, freshly prepared $)$ in the presence of $\mathrm{H}_{2} \mathrm{O}_{2}(312.6 \mathrm{mM})$. To compare the peroxidase-like activity of the AgPt NPs to that of the standard horseradish peroxidase (HRP), the same experiment was done by replacing the AgPt NPs with the same amount of HRP $(0.1 \mathrm{nM})$. Control experiments were carried out where MilliQ water, Ag NPs, and Pt NPs of an equivalent Pt content to that of the AgPt NPs replaced the AgPt NPs. UVvis absorption spectra were recorded with time in the range $300-600 \mathrm{~nm}$ at $37^{\circ} \mathrm{C}$, for a period of $22 \mathrm{~min}$ and the reaction kinetics were determined thereof (Figure S21). The peroxidase-like activity of AgPt NPs was also investigated at different $\mathrm{pH}$ (7 and 11) following the same protocol with replacing the sodium acetate buffer medium with phosphate buffer solutions of $\mathrm{pH} 7$ and 11, respectively (Figure S18). The effect of changing the Ag:Pt molar ratio of the AgPt NPs on the elemental contents of $\mathrm{Ag}$ and $\mathrm{Pt}$ in the resulting $\mathrm{AgPt}$ NPs prepared with $\mathrm{Ag}$ :Pt molar ratios of $(1: 0.0625,1: 0.125,1: 0.25,1: 0.5,1: 0.75,1: 1$ and $1: 1.125$, considering the initial concentrations used during the synthesis) was evaluated by inductively coupled plasma mass spectrometry (ICPMS) using a reported methodology 5 and the peroxidase-like activity of such NPs was investigated following the same protocol mentioned earlier (Figure S19). Also, the peroxidase-like activities of Ag NPs@SC and AgPt NPs@SC were evaluated at different pH (Figure S20) and their kinetics of OPD oxidation are shown in Figure S22. The reaction rates of the peroxidase-like activity of AgPt NPs@BSA were evaluated by plotting the absorbance values at $\lambda=425 \mathrm{~nm}\left(\mathrm{~A}_{425}\right)$ versus the time of the catalytic reaction. To evaluate the steady state kinetics, assays were carried out according to the protocol described above by varying the concentrations of OPD at a fixed concentration of $\mathrm{H}_{2} \mathrm{O}_{2}$ or vice versa (Figures $\mathrm{S} 24$ and $\mathrm{S} 25$ ). 

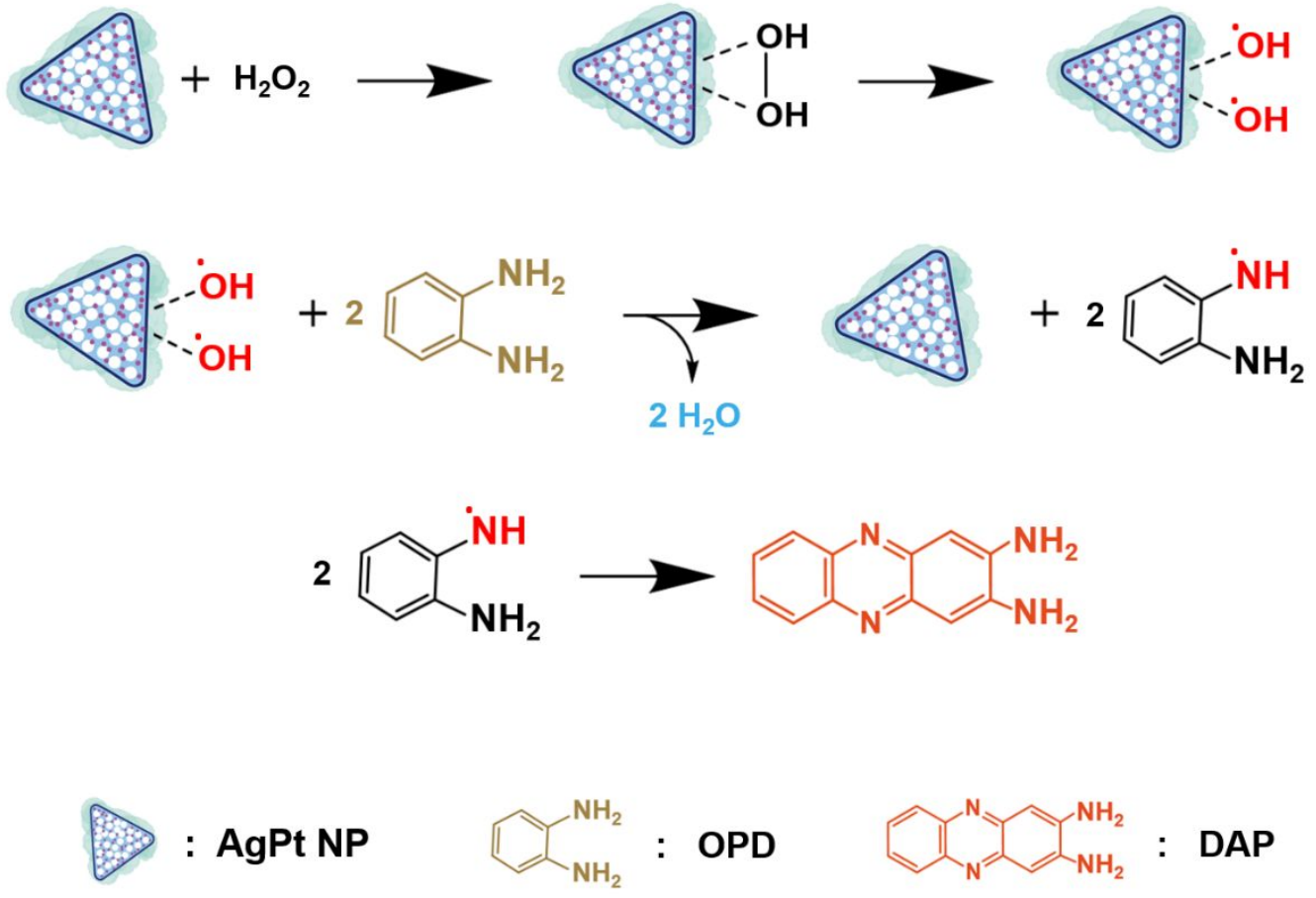

Scheme 1. Assumed mechanism of the peroxidase-like activity of AgPt NPs. OPD was used as the peroxidase substrate. 


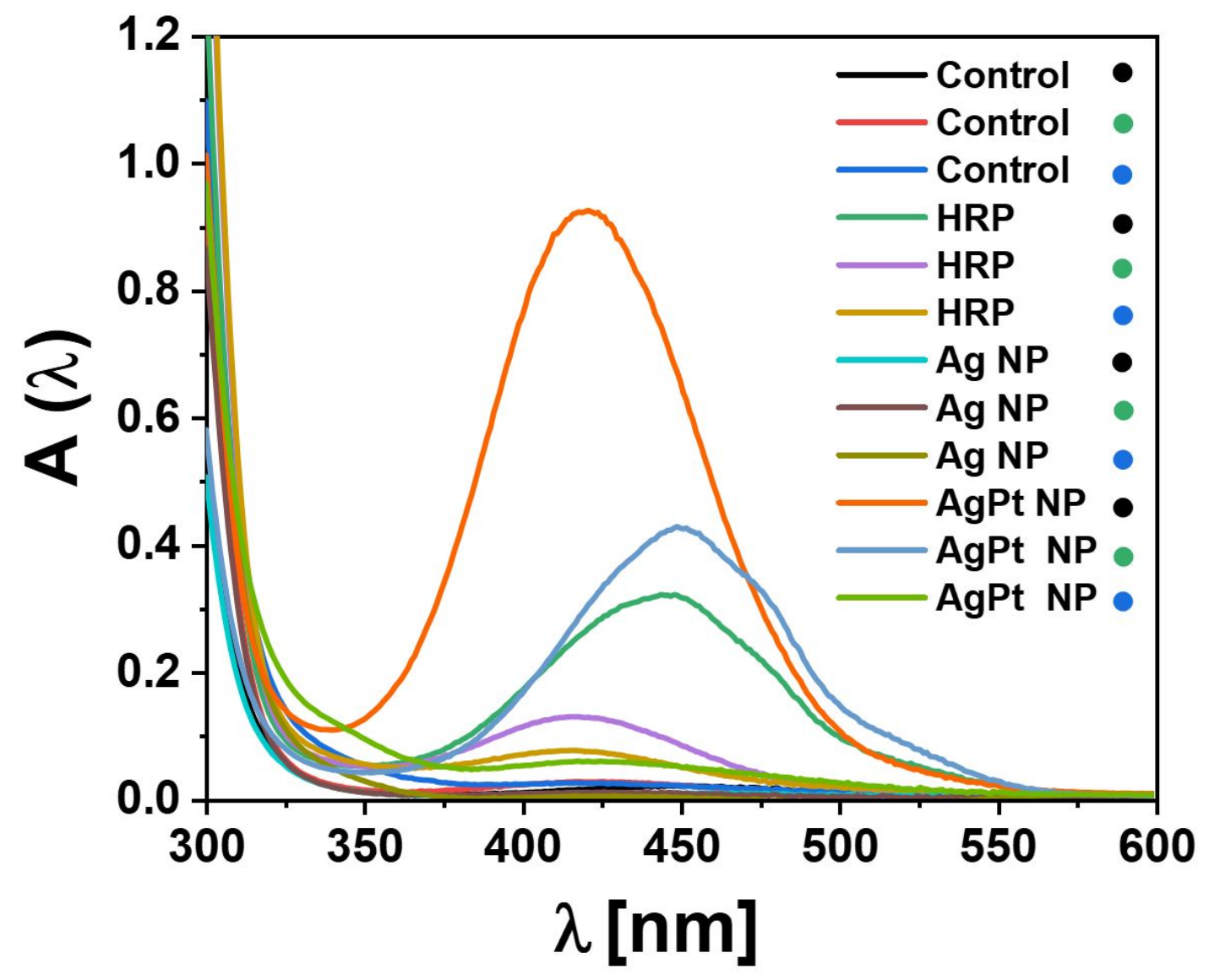

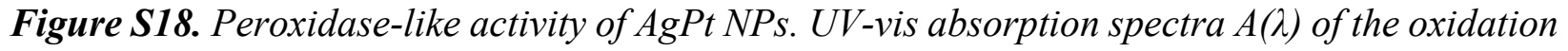
product of OPD by control, HRP, Ag NPs, and AgPt NPs at different $\mathrm{pH}$ ( $\mathrm{pH}$ 4: black circle, $\mathrm{pH}$ 7: green circle and pH 11: blue circle). The AgPt NPs showed the highest peroxidase-like activity at $\mathrm{pH} 4$. 

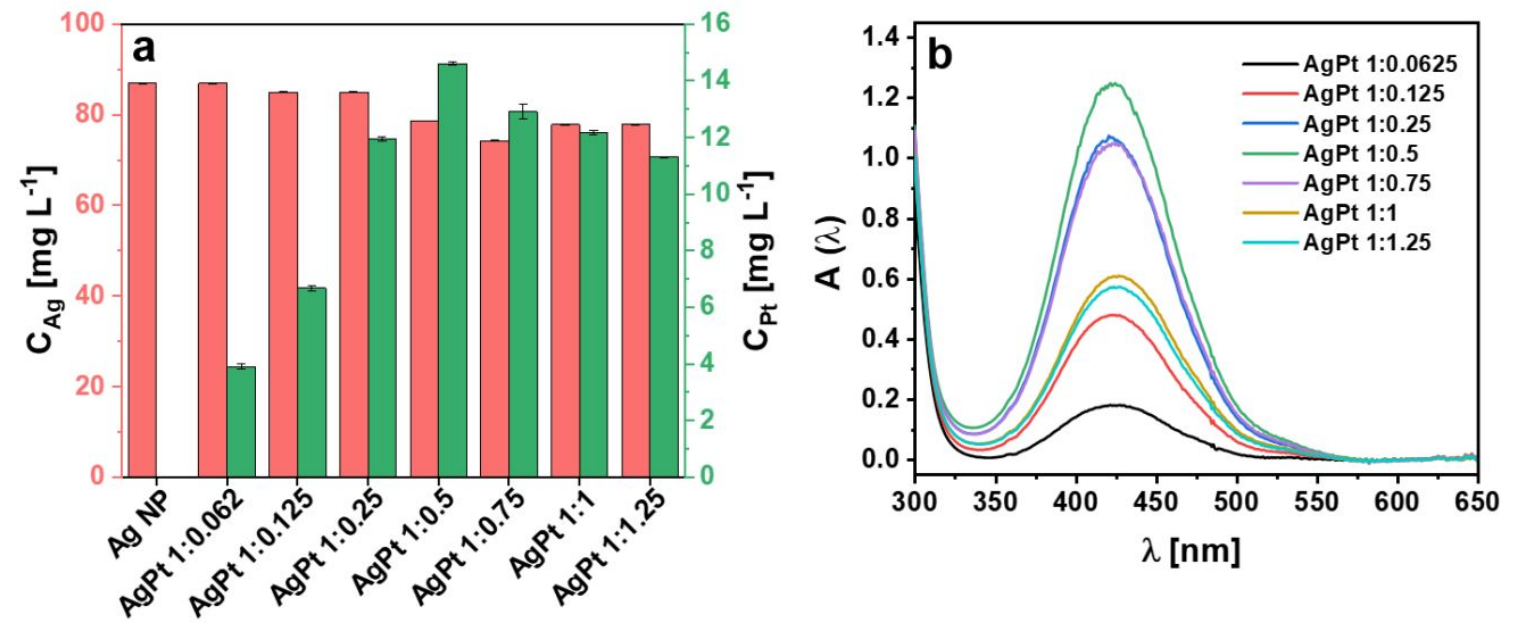

Figure S19. Intrinsic peroxidase-like activity of AgPt NPs as a function of their elemental composition. (a) ICP-MS analysis of AgPt NPs prepared using different molar ratios of Ag to Pt $\left(C_{A g}\right.$ and $C_{P t}$ refer to the mass concentrations) and (b) their corresponding peroxidase-like activity as shown from the UV-vis absorption spectra $A(\lambda)$ of the OPD oxidation product at $\mathrm{pH} 4$. 


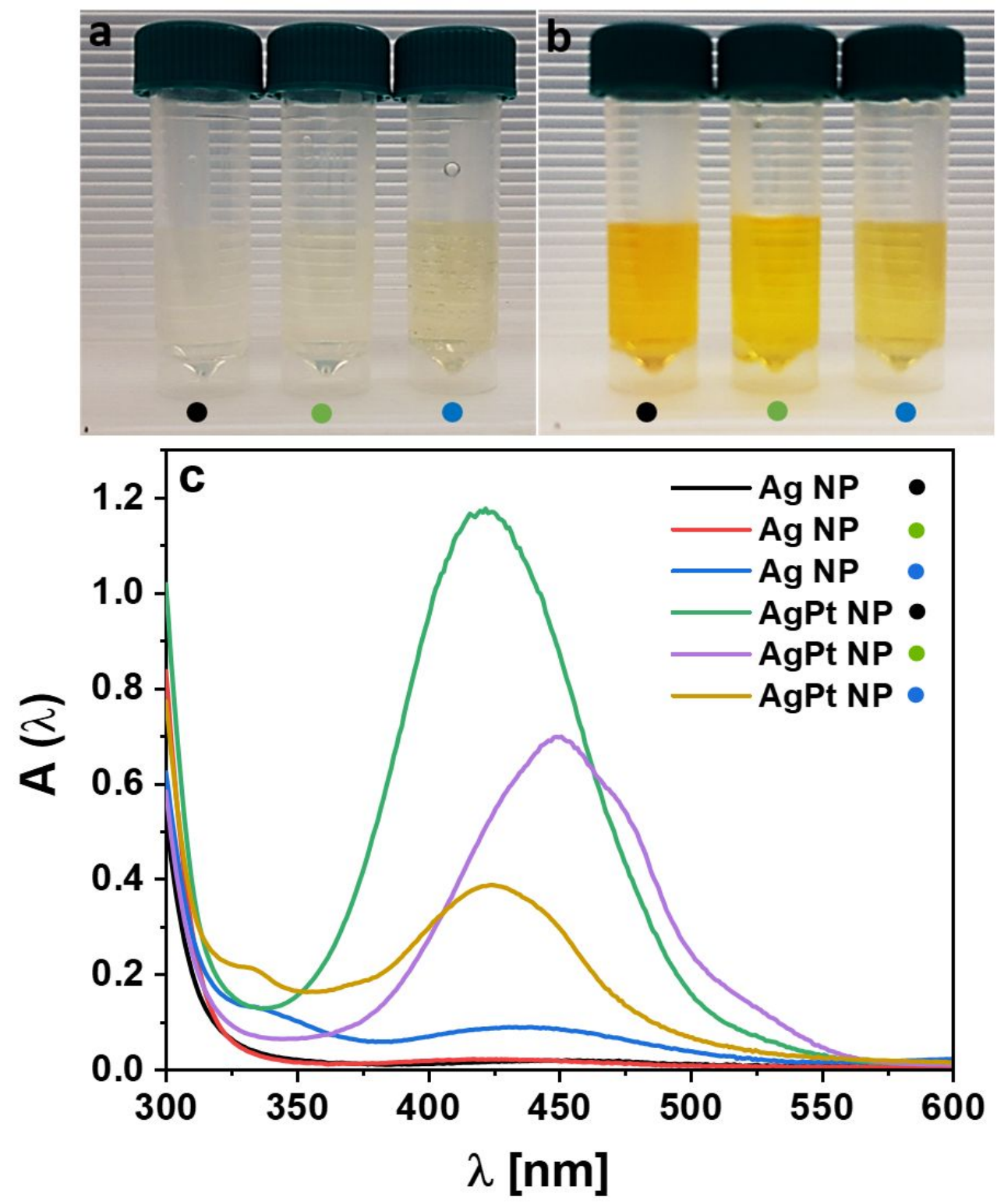

Figure S20. Photographs of the peroxidase-like activity of (a)AgNP@SC and (b)AgPt NPs@SC at different $\mathrm{pH}$ ( $\mathrm{pH}$ 4: black circle, $\mathrm{pH}$ 7: green circle and $\mathrm{pH}$ 11: blue circle) and (c) their corresponding $U V$-vis absorption spectra $A(\lambda)$. 

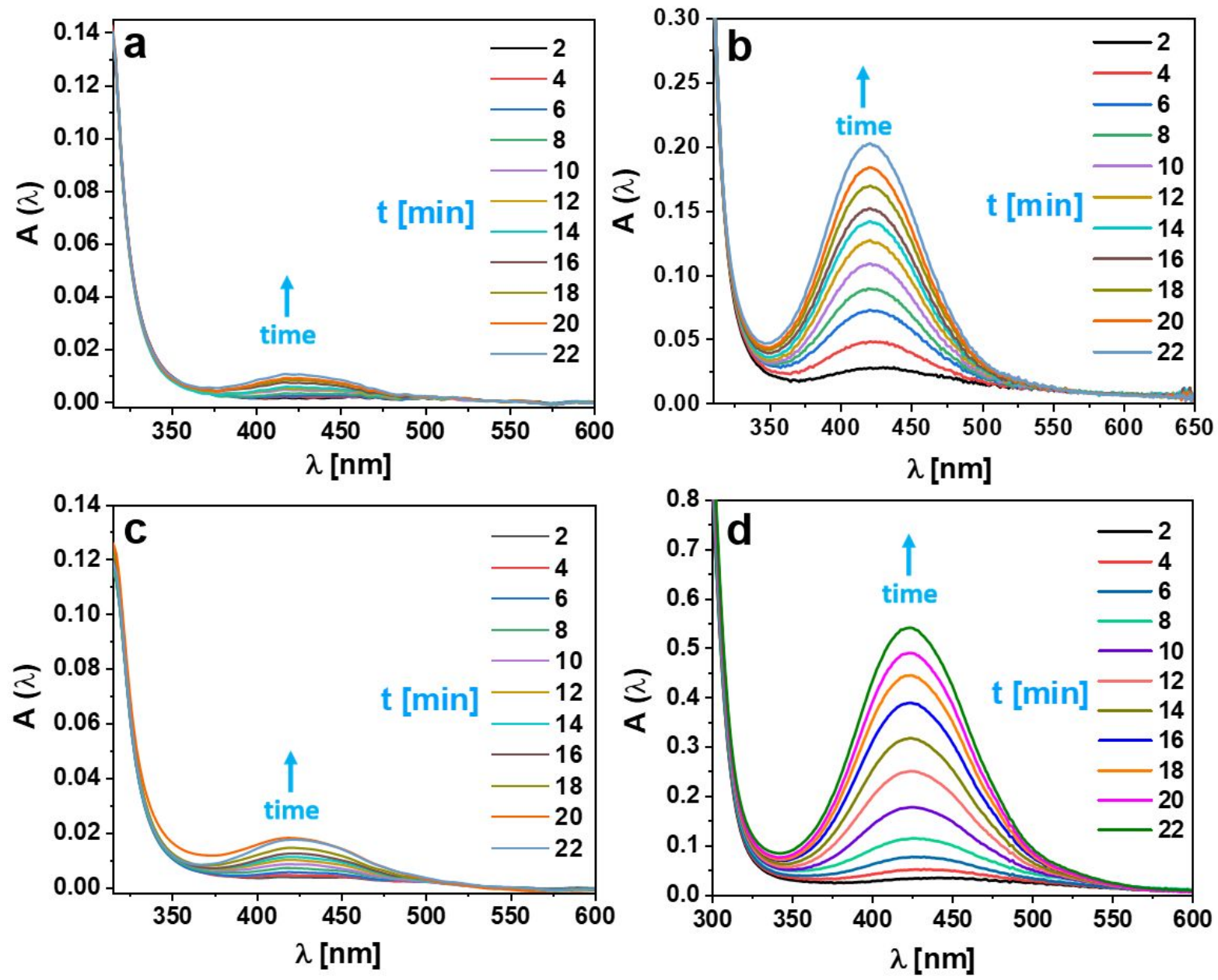

Figure S21. Kinetics of OPD oxidation. Time-dependent absorbance spectra $A(\lambda)$ of the oxidized OPD (DAP) generated upon oxidation of OPD (a) in absence of catalyst, (b) in presence of HRP, (c) Ag NPs, or (d) AgPt NPs at pH 4. 

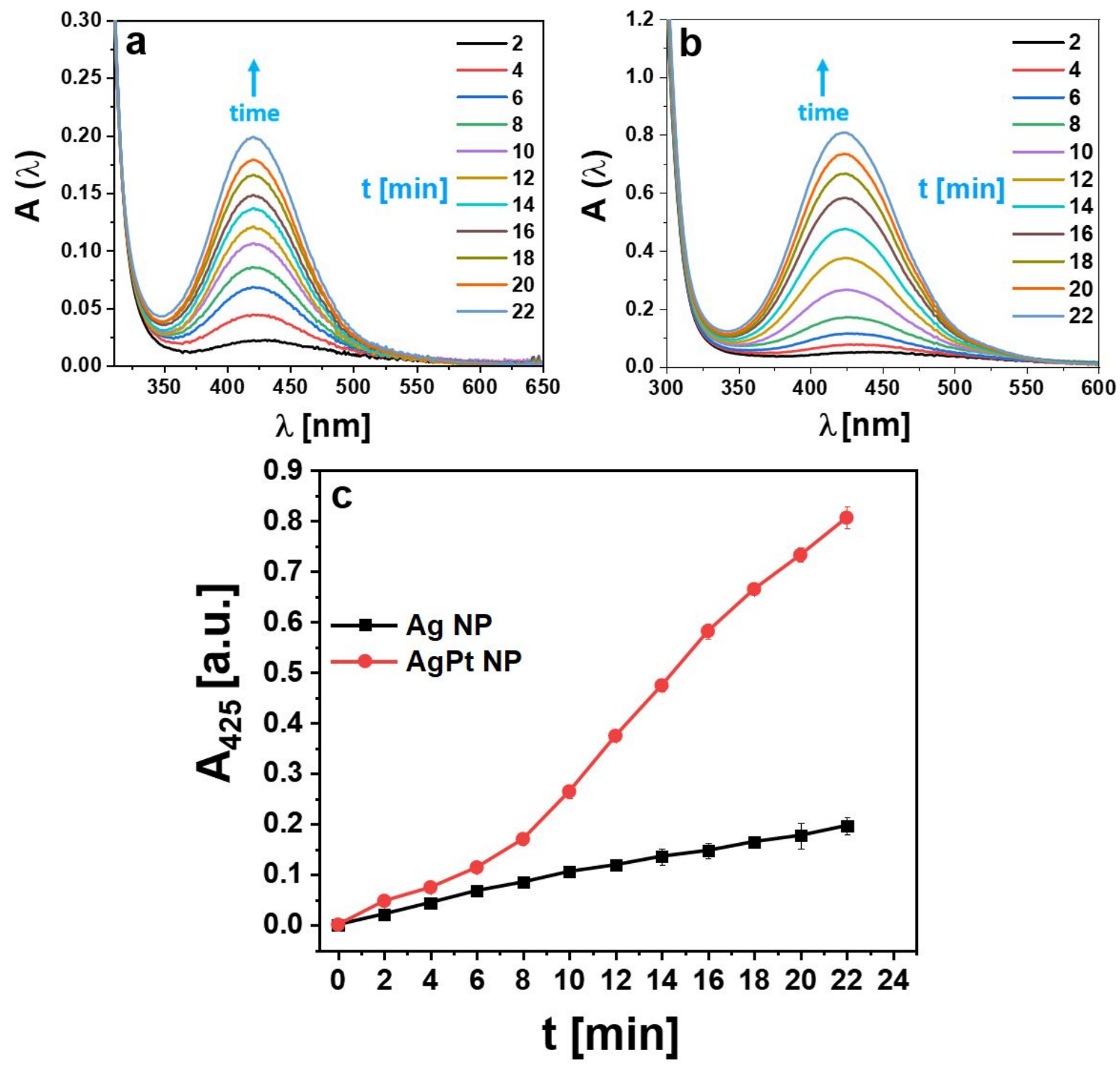

Figure S22. Kinetics of OPD oxidation. Time-dependent absorbance spectra $A(\lambda)$ of the oxidized OPD (DAP) generated upon oxidation of OPD in presence of (a) Ag NPs@SC and (b) AgPt NPs@SC at pH 4. (c) Time-dependent absorbance changes at $\lambda=425 \mathrm{~nm}$ upon oxidation of OPD to DAP in presence of Ag NP@SC or AgPt NPs@SC. 

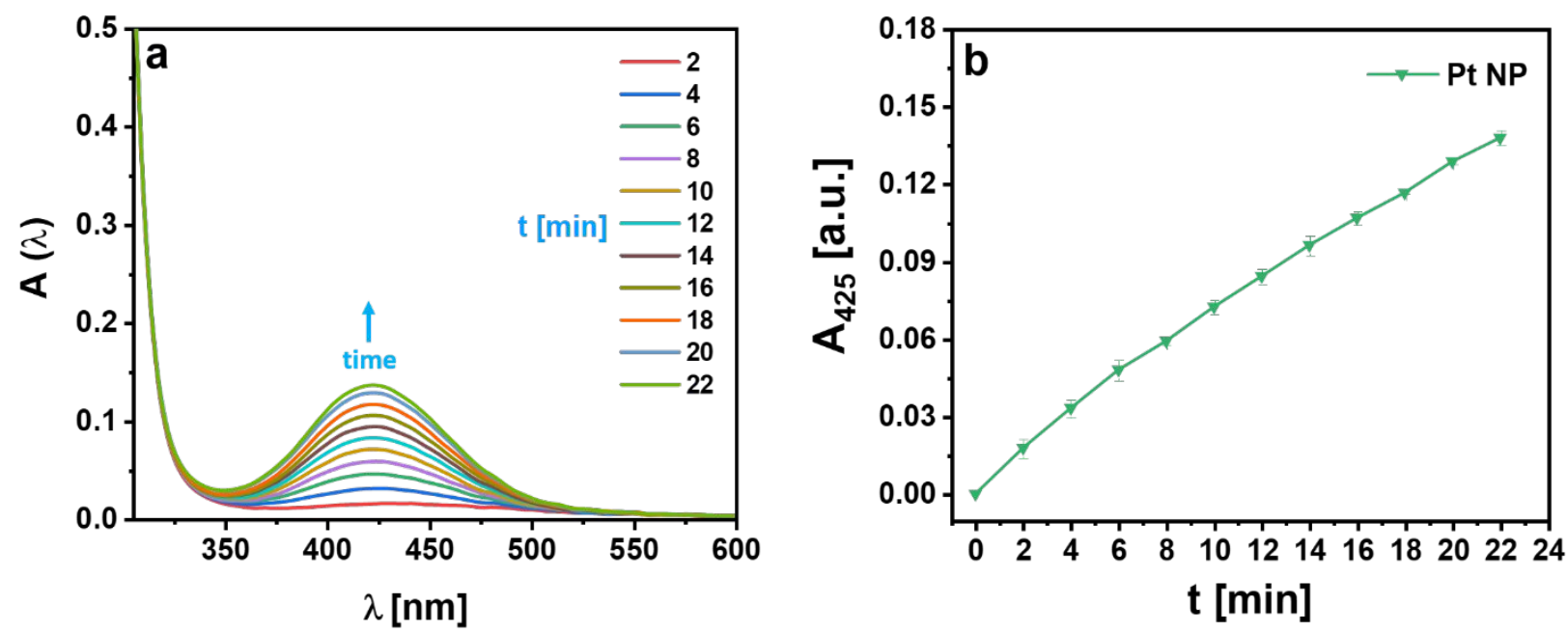

Figure S23. Peroxidase-like activity of Pt NPs@SC. (a) Time-dependent changes in the UV-vis absorption spectra $A(\lambda)$ of the oxidation product of OPD by Pt NPs@SC at pH 4. (b) Timedependent absorbance changes at wavelength $\lambda=425 \mathrm{~nm}$ upon oxidation of OPD to DAP in presence of PtNPs@SC atpH4. 

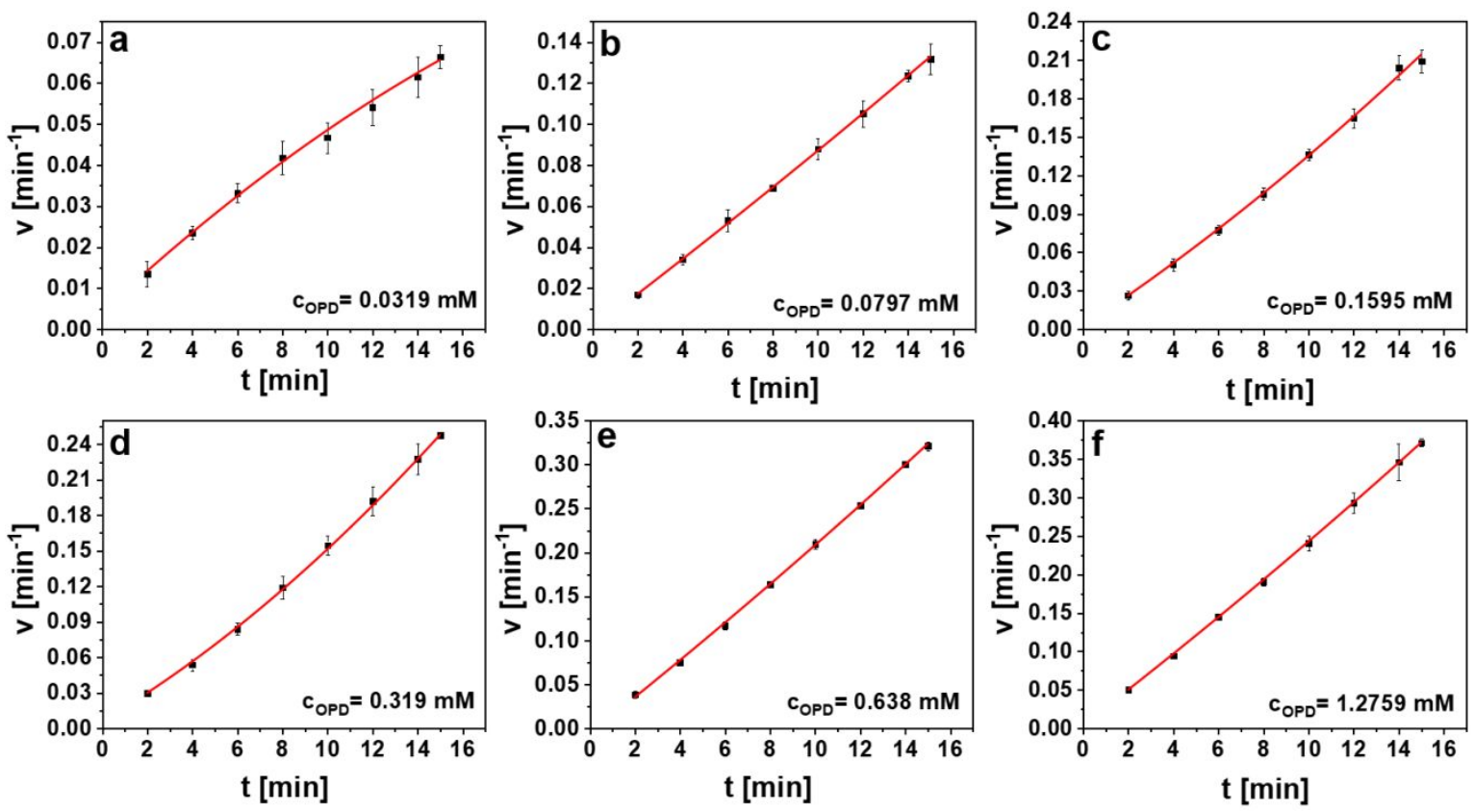

Figure S24. Apparent kinetics of AgPt NPS as peroxidase mimics. (a-f) Rate of the reaction (v)time course curves of OPD oxidation using different concentrations of OPD $\left(c_{O P D}=0.0319\right.$, 0.0797, 0.1595, 0.319, 0.638 and $1.2759 \mathrm{mM}$, respectively) while keeping the $\mathrm{H}_{2} \mathrm{O}_{2}$ concentration fixed. 

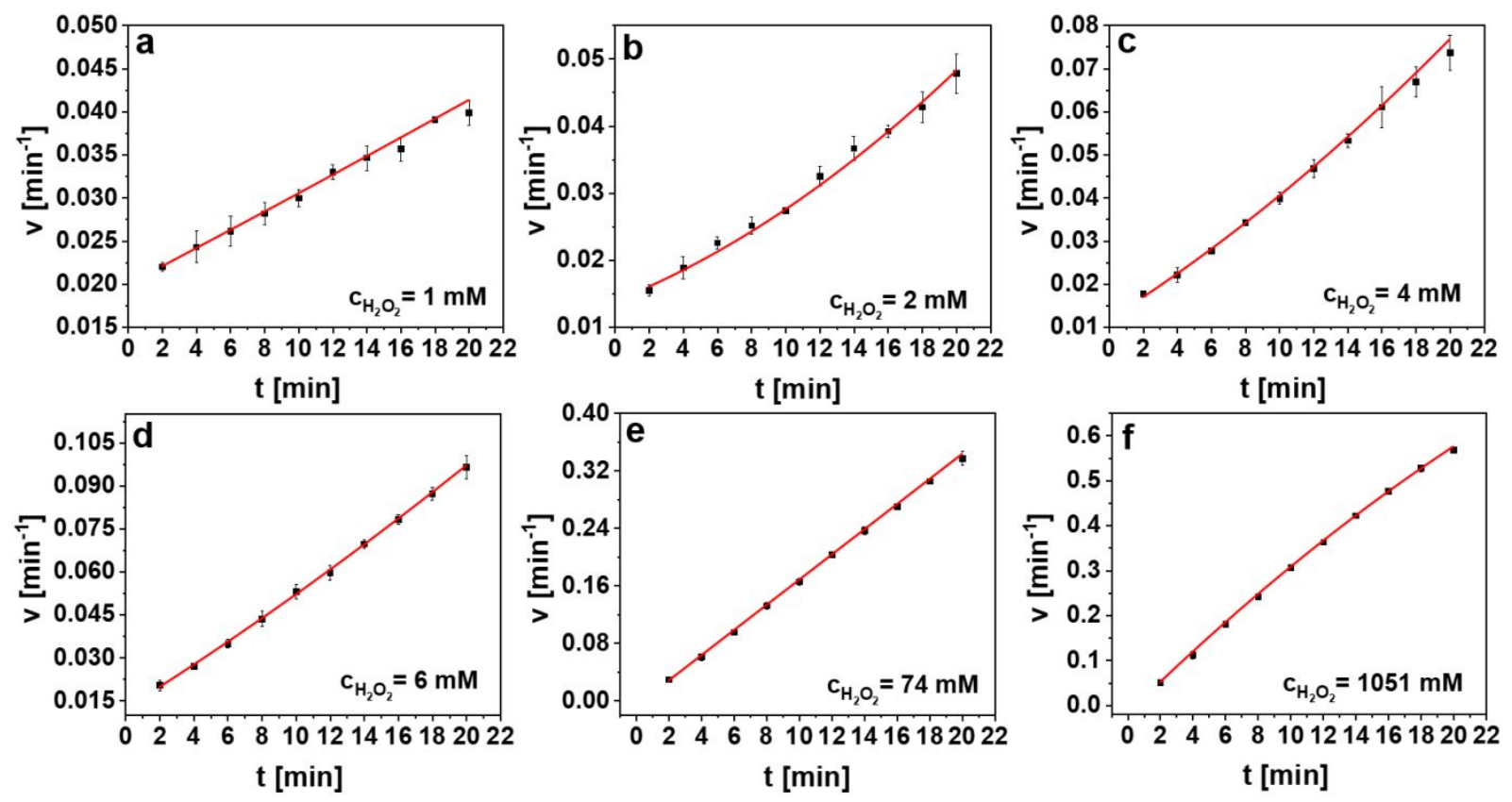

Figure S25. Apparent kinetics of AgPt NPs as peroxidase mimics. (a-f) Rate of the reaction (v)time course curves of OPD oxidation using different concentrations of $\mathrm{H}_{2} \mathrm{O}_{2}\left(c_{\mathrm{H}_{2} \mathrm{O}_{2}}=1,2,4,6\right.$, 74 and $1051 \mathrm{mM}$, respectively) while keeping the OPD concentration fixed. 


\section{Catalase-like activity of AgPt NPs}

The catalase-like activity of AgPt NPs was evaluated by monitoring the generation of $\mathrm{O}_{2}$ as a result of $\mathrm{H}_{2} \mathrm{O}_{2}$ decomposition using an Extech DO210 model dissolved oxygen meter. In a typical experiment, the catalase-like activity assay was performed at room temperature by mixing $1 \mathrm{~mL}$ of AgPt NPs (Final concentration of NPs $\left(c_{N P}\right)=0.0332 \mathrm{nM}$ ) in a reaction volume of $5 \mathrm{~mL}$ phosphate buffer solution $(\mathrm{pH}=11)$ in the presence of $\mathrm{H}_{2} \mathrm{O}_{2}(750 \mathrm{mM})$. To compare the catalaselike activity of the AgPt NPs to that of the standard catalase, the same experiment was done by replacing the AgPt NPs with the same amount of catalase $(20 \mathrm{U} / \mathrm{mL} ; 1 \mathrm{U}(\mu \mathrm{mol} / \mathrm{min})$ is defined as the amount of the enzyme that catalyzes the conversion of one micromole of substrate per minute under the specified conditions of the assay method). Control experiments were carried out where MilliQ water, Ag NPs, and Pt NPs of an equivalent Pt content to that of the AgPt NPs replaced the AgPt NPs. Air bubbles were observed several minutes after the addition of $\mathrm{H}_{2} \mathrm{O}_{2}$, and dissolved oxygen levels were recorded using the oxygen meter over a period of 10 minutes and the reaction rates of the catalse-like activity of were evaluated by plotting concentrations of the liberated oxygen $\left(\mathrm{C}_{\mathrm{O}_{2}}\right)$ versus the time of the catalytic reaction (Figure 3e). The catalase-like activity of $\mathrm{AgPt}$ NPs was also investigated at different $\mathrm{pH}$ (4 and 7) following the same protocol with replacing the phosphate buffer solution of $\mathrm{pH} 11$ with sodium acetate buffer of $\mathrm{pH} 4$ and phosphate buffer solutions of pH 7, respectively (Figure 3 a-d). The catalase-like activities of Ag NPs@SC and AgPt NPs@SC were evaluated at different pH (Figure S26 a and b). The effect of changing the Ag:Pt molar ratio of the AgPt NPs on the catalase-like activity was evaluated for AgPt NPs with molar ratios of $(1: 0.0625,1: 0.125,1: 0.25,1: 0.5,1: 0.75,1: 1$ and 1:1.125, considering the initial concentrations used during the synthesis) following the same protocol mentioned above (Figure 3f). To evaluate the steady state kinetics, assays were carried out according to the protocol described above by varying the concentrations of $\mathrm{H}_{2} \mathrm{O}_{2}$ and recording the rate of the catalytic reaction (Figure S28). 


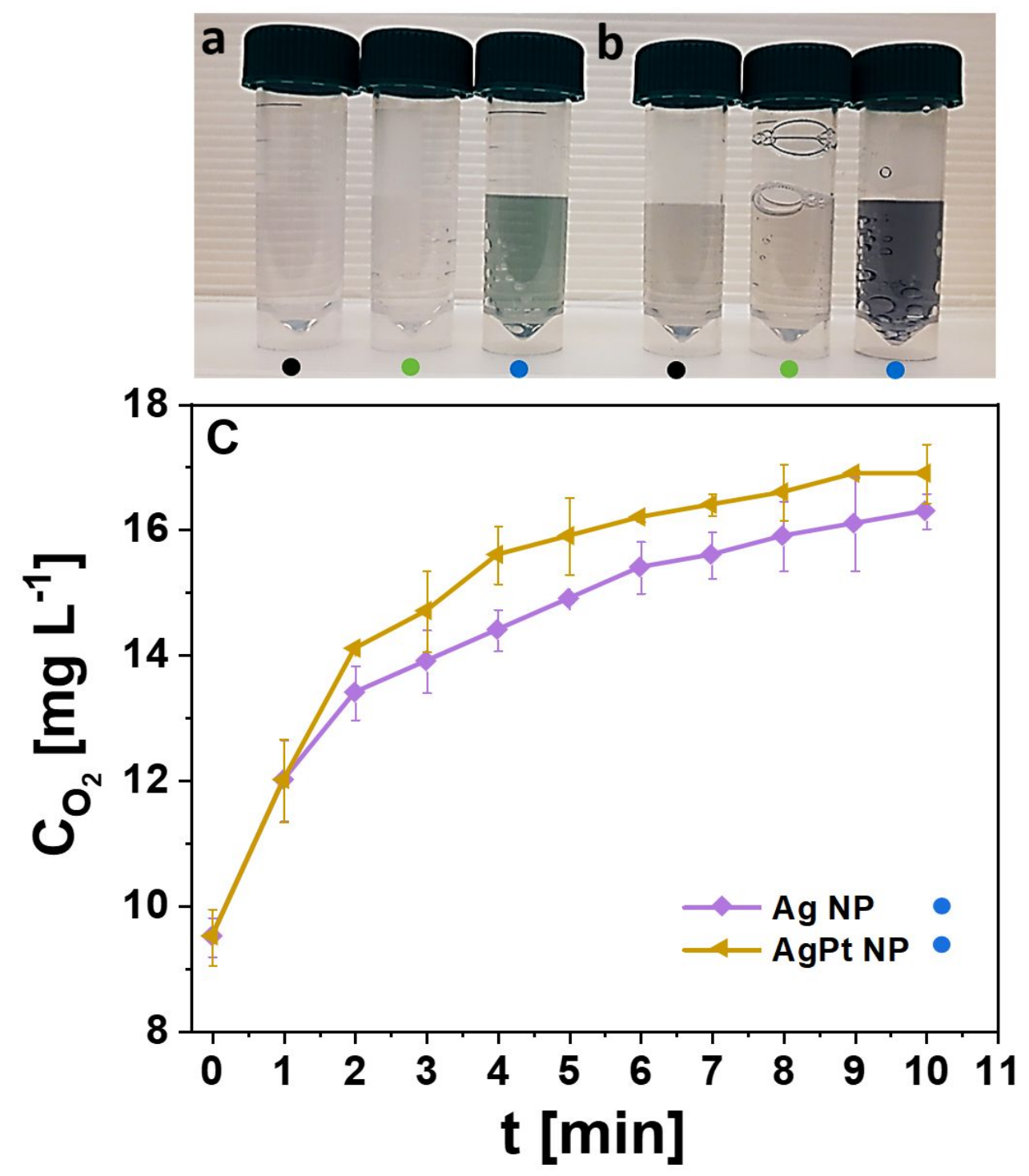

Figure S26. Catalase-like activity of AgNPs@SC and AgPt NPs@SC.Photographs of catalaselike activity of (a)Ag NPs@SC and (b) AgPt NPs@SC at differentpH (pH 4: black circle,pH 7: green circle and pH 11: blue circle). (c) Time-dependent changes in the concentration of liberated $\mathrm{O}_{2}\left(\mathrm{C}_{\mathrm{O}_{2}}\right)$ during the catalytic decomposition of $\mathrm{H}_{2} \mathrm{O}_{2}$ in presence of AgNPs@SCorAgPt NPs@SC at $\mathrm{pH} 11$. 


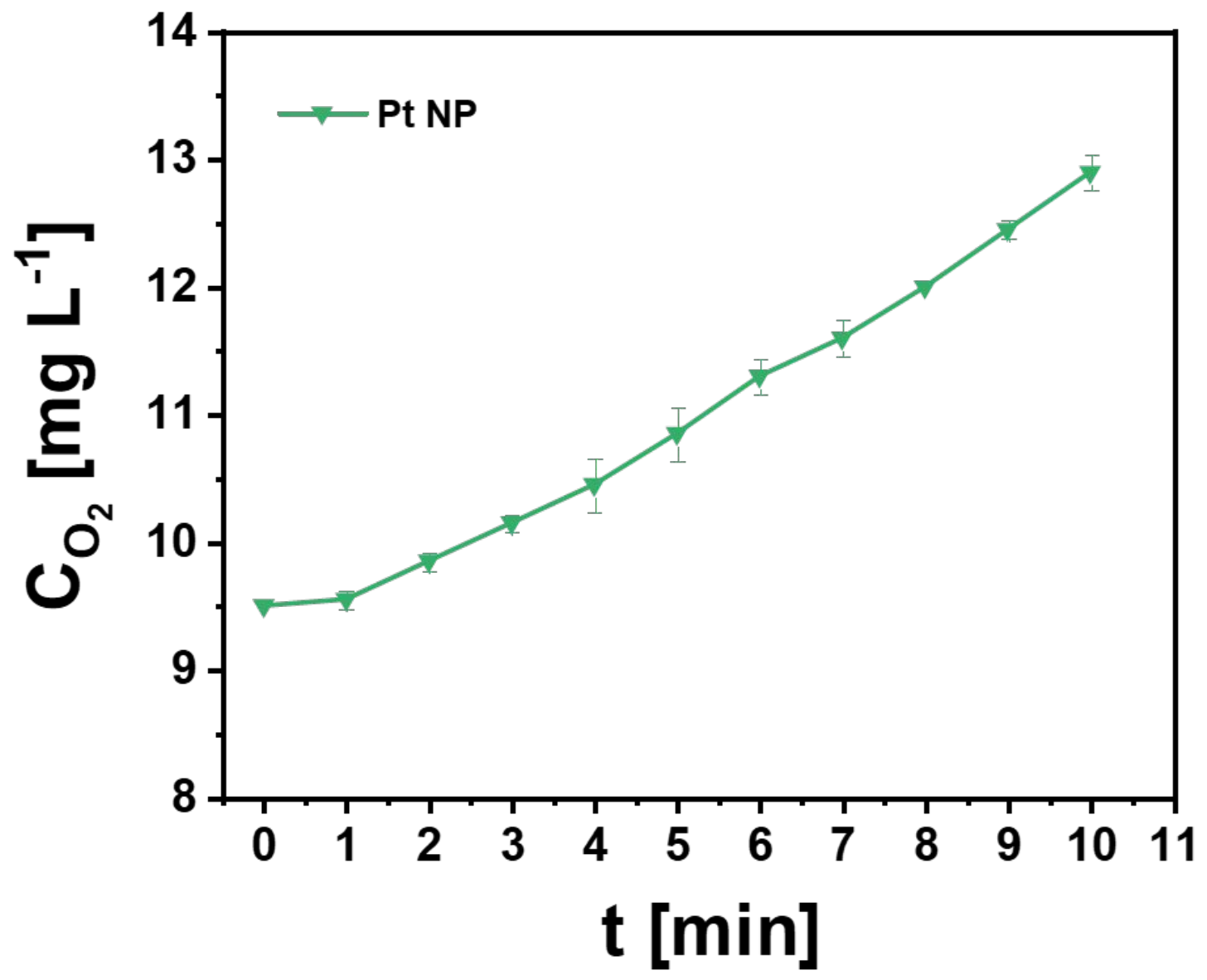

Figure S27. Catalase-like activity of Pt NPs@SC. Time-dependent changes in the concentration of liberated $\mathrm{O}_{2}\left(\mathrm{C}_{\mathrm{O}_{2}}\right)$ during the catalytic decomposition of $\mathrm{H}_{2} \mathrm{O}_{2}$ in presence of Pt NPs@SC at pH 11 . 

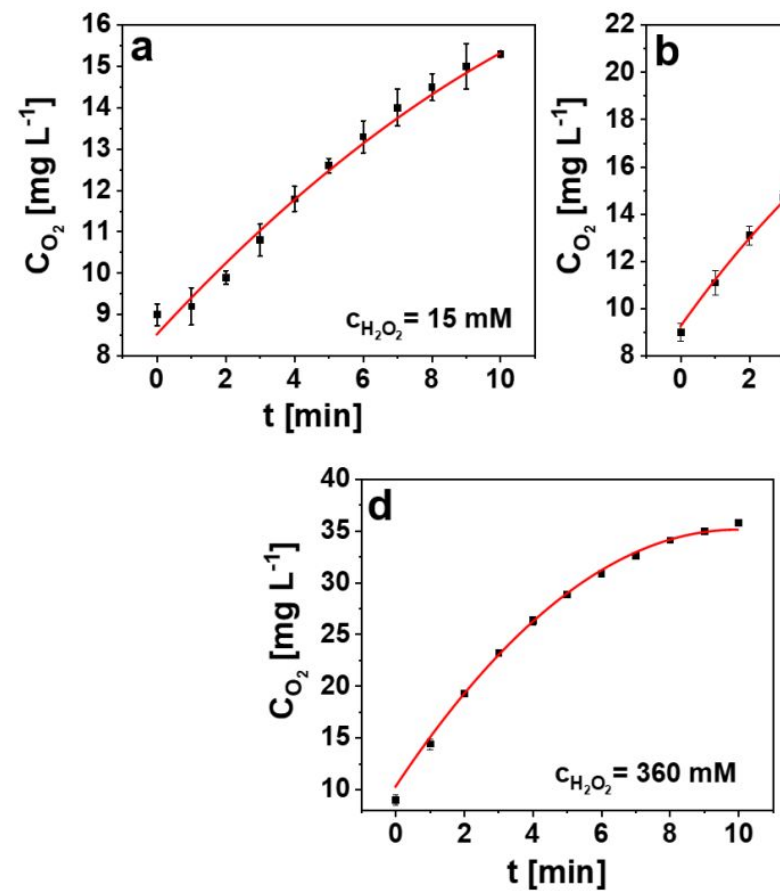
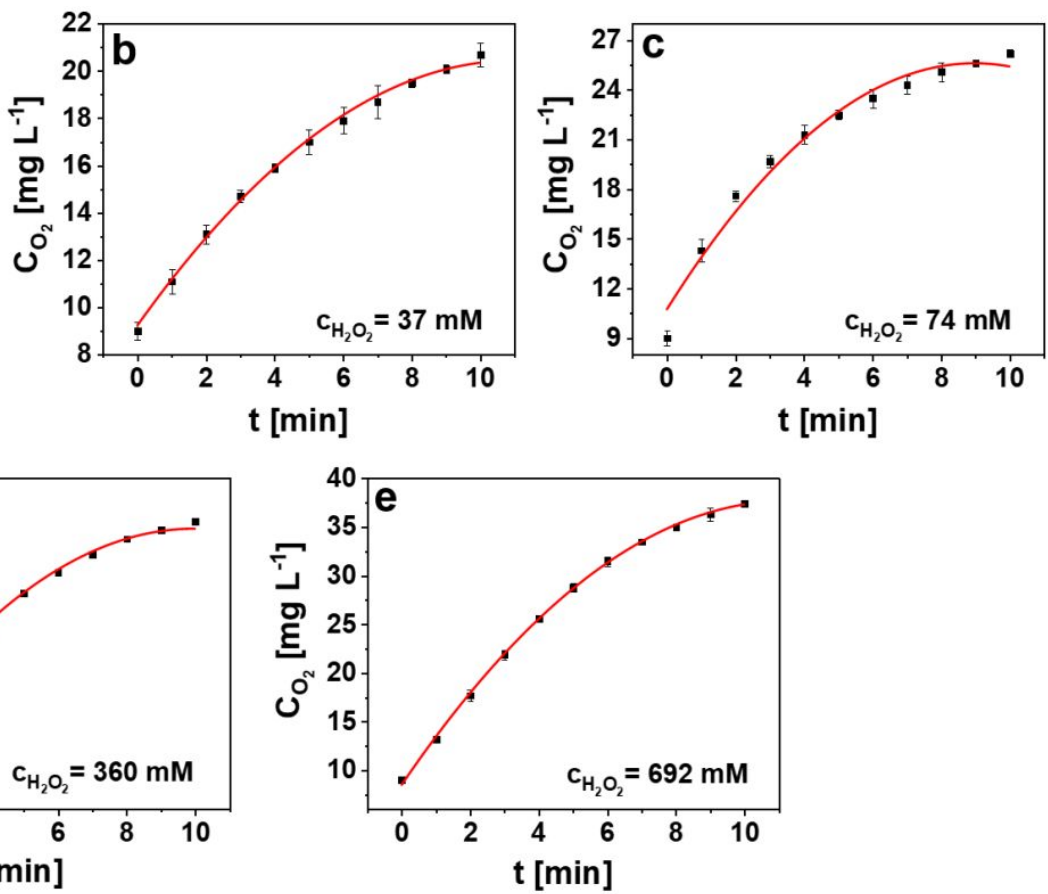

Figure S28. Apparent kinetics of AgPt NPs as catalase mimics. (a-e) Oxygen evolution-time course curves of $\mathrm{H}_{2} \mathrm{O}_{2}$ decomposition by AgPt NPs using different concentrations of $\mathrm{H}_{2} \mathrm{O}_{2}\left(\mathrm{C}_{\mathrm{H}_{2} \mathrm{O}_{2}}=\right.$ 15, 37, 74, 360 and $692 \mathrm{mM}$, respectively). 


\section{Cargo-loading/-controlled release into/from the AgPt NPs}

In order to evaluate the cargo-loading ability of the porous AgPt NPs, the hydrophilic fluorescent dye calcein was used as model drug. In a typical experiment, ten milliliters of the as-synthesized Ag NPs and AgPt NPs solutions were transferred to $20 \mathrm{~mL}$ glass vials and were allowed to react with $1.5 \mathrm{~mL}$ calcein solution of different concentrations so that to bring the final calcein concentration in the reaction mixture to $0.15,0.3,0.6,0.75,1.2,1.5,2.25,3,4.5,7.5$, and $15 \mu \mathrm{M}$. After incubation for $60 \mathrm{~min}$, free calcein dye was removed by multiple cycles of centrifugation and washing, until no calcein was detectable in the supernatants either spectrophotometrically or by measuring the fluorescence of the supernatants. Then, the absorption and fluorescence spectra $\left(\lambda_{\mathrm{ex}}=490 \mathrm{~nm}\right.$ and $\lambda_{\mathrm{em}}=515 \mathrm{~nm}$ ) of free un-incorporated calcein dye in the supernatants were recorded and the calcein concentration in these supernatant was evaluated thereafter (Figures S30 and $4 \mathrm{~b}$ ) using a calibration curve (Figure S29). Then, AgPt NPs were incubated with different amounts of calcein $(1.2,1.5,3,4.5,9,22,44,72$, and $139 \mu \mathrm{M})$ to find out the loading content (LC) (mg calcein/mg AgPt NPs) and \% loading efficiency (LE) of calcein dye into the AgPt NPs (Figures 4c and S31) according to the following equations:

$$
\begin{aligned}
& \text { Calcein loading content }=\frac{\text { Weight of entrapped calcein }}{\text { Weight of NPs }} \\
& \% \text { Loading efficiency }=\frac{\text { Weight of entrapped calcein }}{\text { Total weight of calcein added }} \times 100
\end{aligned}
$$

The controlled calcein release experiments were performed by recording the fluorescence intensity of calcein-loaded AgPt NPs upon the addition of the triggering agent, $\mathrm{H}_{2} \mathrm{O}_{2}$. In a typical release experiment, to $1 \mathrm{~mL}$ of calcein-loaded AgPt NPs (or calcein-loaded Ag NPs for comparison) 20 $\mu \mathrm{L}$ of $30 \% \mathrm{H}_{2} \mathrm{O}_{2}$ was added. Immediately after the triggering agent was added, the fluorescence intensity at $\lambda_{\mathrm{em}}=515 \mathrm{~nm}$ was recorded (Figure $4 \mathrm{~d}$ and e). The same experiment was repeated using AgPt NPs loaded with different calcein concentrations and the fluorescence intensities were recorded at $\lambda_{\mathrm{em}}=515$ (Figure $\mathrm{S} 32$ ). 

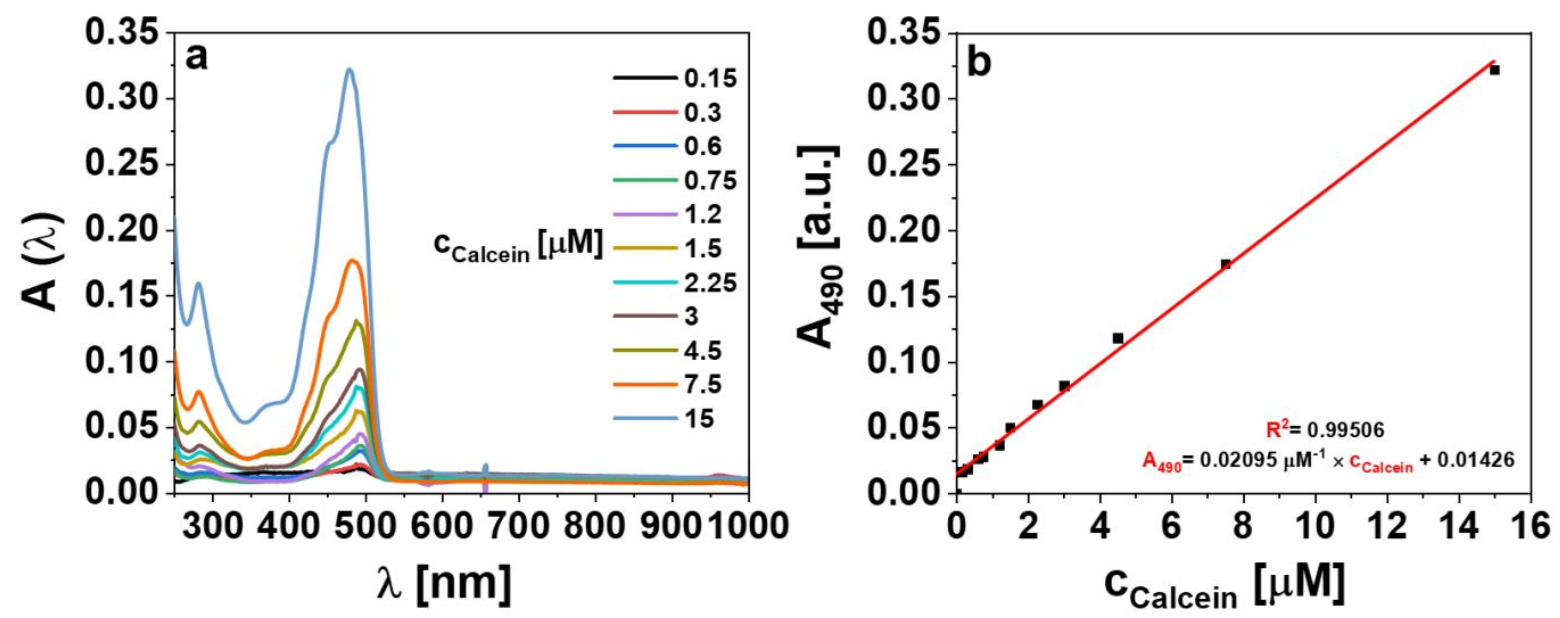

Figure S29. Calcein standard curve. (a) UV-vis absorption spectra $A(\lambda)$ of different calcein concentrations and (b) calibration curve of calcein plotted by measuring the absorbance of different calcein concentrations in (a) at $\lambda=490 \mathrm{~nm} . R^{2}$ indicates how well the data points are fitted by the linear regression line.
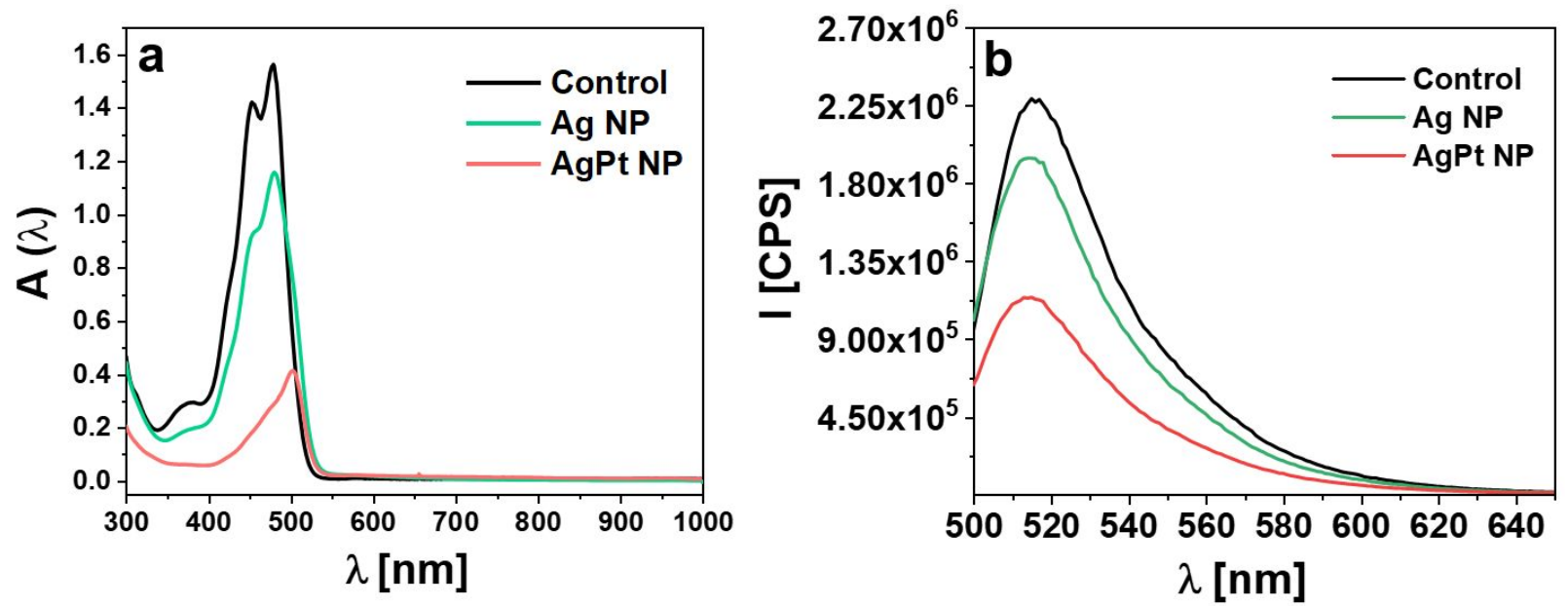

Figure S30. (a) UV-vis absorption spectra $A(\lambda)$ and (b) emission spectra of the supernatants after reaction of $A g N P s$ and AgPt NPs with calcein, respectively, relative to control calcein solution. $C P S=$ counts per second. 

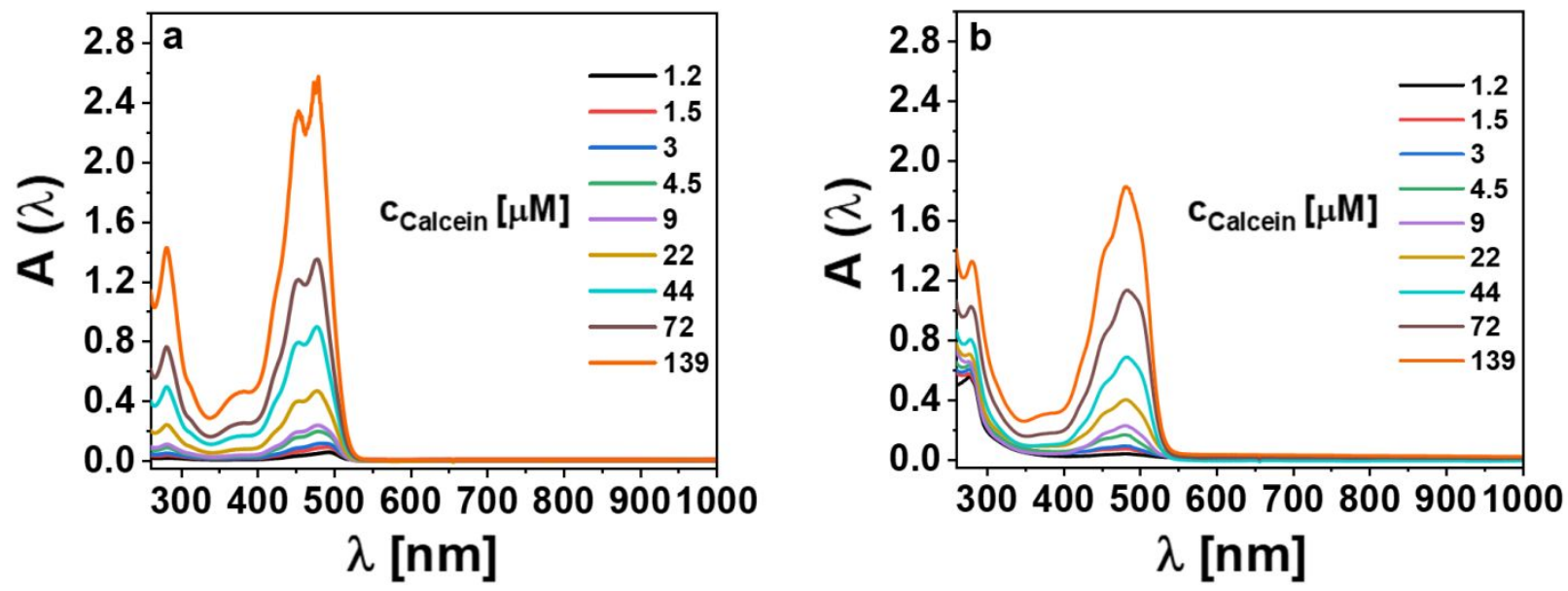

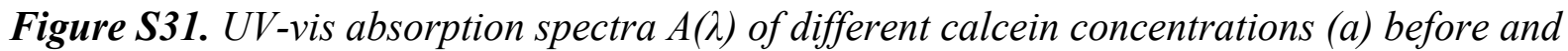
(b) after reacting with AgPt NPs.
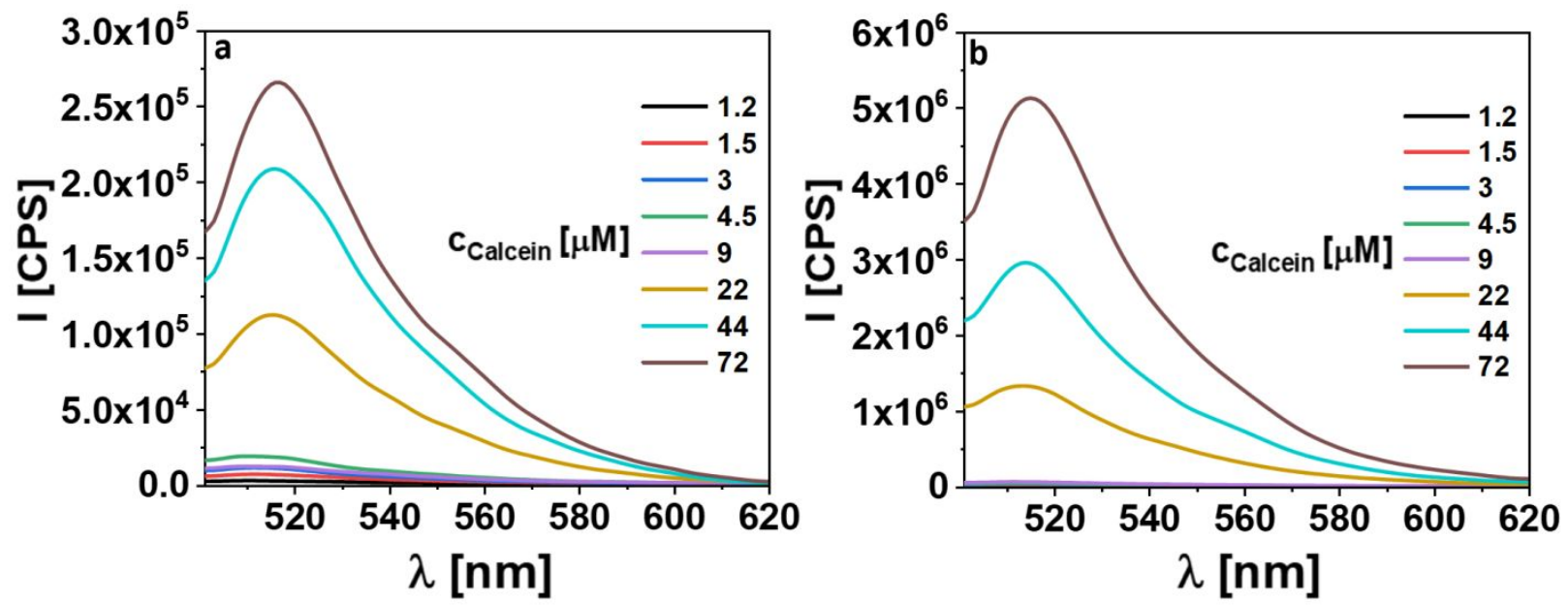

Figure S32. Controlled release of calcein after loading AgPt NPs with different concentrations of calcein. Emission spectra of calcein (a) before and (b) after the addition of exogenous stimulus $\left(\mathrm{H}_{2} \mathrm{O}_{2}\right)$ to the calcein-loaded AgPt NPs. 


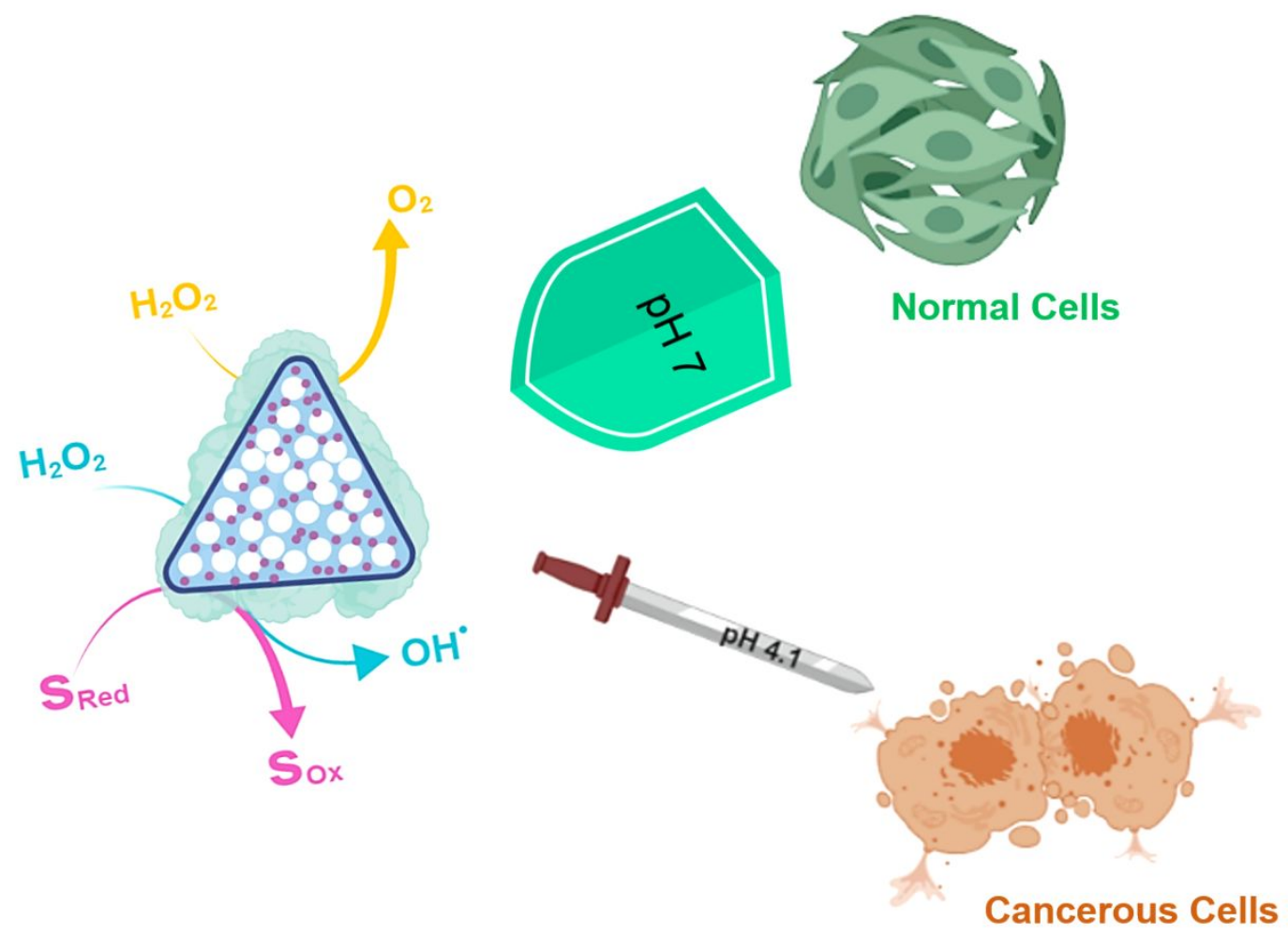

Figure S33. Sword and shield hypothesis and the selectivity of AgPt NPs in different pH environments. 


\section{References}

1. Chakraborty, I.; Feliu, N.; Roy, S.; Dawson, K.; Parak, W. J., Protein-Mediated ShapeControl of Silver Nanoparticles. Bioconjugate Chem. 2018, 29, 1261-1265.

2. Jin, R.; Cao, Y. C.; Hao, E.; Metraux, G. S.; Schatz, G. C.; Mirkin, C. A., Controlling Anisotropic Nanoparticle Growth through Plasmon Excitation. Nature 2003, 425, 487-490.

3. Stierle, A.; Keller, F. T.; Noei, H.; Vonk, V.; Roehlsberger, R., Desy Nanolab. Journal of large-scale research facilities 2016, 2, A76.

4. Feliu, N.; Pelaz, B.; Zhang, Q.; del_Pino, P.; Nyström, A.; Parak, W. J., Nanoparticle Dosage-a Nontrivial Task of Utmost Importance for Quantitative Nanosafety Research. Wiley Interdiscip Rev Nanomed Nanobiotechnol 2016, 8, 479-492.

5. Hühn, J., et al., Selected Standard Protocols for the Synthesis, Phase Transfer, and Characterization of Inorganic Colloidal Nanoparticles. Chem. Mater. 2017, 29, 399-461.

6. Weisstein, E. W. Heron's Formula. http://mathworld.wolfram.com/HeronsFormula.html.

7. Xia, X.; Wang, Y.; Ruditskiy, A.; Xia, Y., 25th Anniversary Article: Galvanic Replacement: A Simple and Versatile Route to Hollow Nanostructures with Tunable and Well-Controlled Properties. Adv. Mater. 2013, 25, 6313-6333.

8. Micsonai, A.; Wien, F.; Bulyaki, E.; Kun, J.; Moussong, E.; Lee, Y.-H.; Goto, Y.; Refregiers, M.; Kardos, J., Bestsel: A Web Server for Accurate Protein Secondary Structure Prediction and Fold Recognition from the Circular Dichroism Spectra. Nucleic Acids Res. 2018, 46, 315-322.

9. Gharib, M.; Khalaf, M.; Afroz, S.; Feliu, N.; Parak, W. J.; Chakraborty, I., Sustainable Synthesis and Improved Colloidal Stability of Popcorn-Shaped Gold Nanoparticles. ACS Sustainable Chemistry \& Engineering 2019, 7, 9834-9841.

10. Halfman, C. J.; Nishida, T., Influence of Ph and Electrolyte on the Fluorescence of Bovine Serum Albumin. Biochimica et Biophysica Acta (BBA)-Protein Structure 1971, 243, 284-293.

11. Halfman, C. J.; Nishida, T., Nature of the Alteration of the Fluorescence Spectrum of Bovine Serum Albumin Produced by the Binding of Dodecyl Sulfate. Biochimica et Biophysica Acta (BBA)-Protein Structure 1971, 243, 294-303.

12. Steinhardt, J.; Krijn, J.; Leidy, J. G., Differences between Bovine and Human Serum Albumins. Binding Isotherms, Optical Rotatory Dispersion, Viscosity, Hydrogen Ion Titration, and Fluorescence Effects. Biochemistry 1971, 10, 4005-4015.

13. Cowgill, R. W., Fluorescence and Protein Structure: Xiv. Tyrosine Fluorescence in Helical Muscle Proteins. Biochimica et Biophysica Acta (BBA)-Protein Structure 1968, 168, 417-430. 